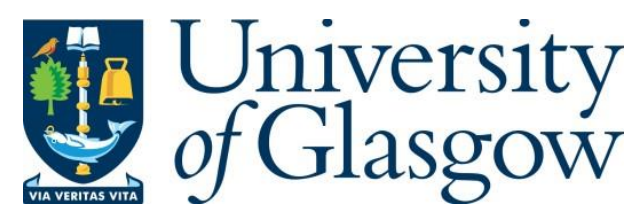

Levy, Y. (2013) Discounted stochastic games with no stationary Nash equilibrium: two examples. Econometrica, 81(5), pp. 1973-2007.

There may be differences between this version and the published version. You are advised to consult the publisher's version if you wish to cite from it.

This is the peer reviewed version of the following article Levy, Y. (2013)

Discounted stochastic games with no stationary Nash equilibrium: two

examples. Econometrica, 81(5), pp. 1973-2007, which has been published in final form at http://dx.doi.org/10.3982/ECTA10530

This article may be used for non-commercial purposes in accordance with Wiley Terms and Conditions for Self-Archiving.

http://eprints.gla.ac.uk/129890/

Deposited on: 08 November 2019

Enlighten - Research publications by members of the University of Glasgow http://eprints.gla.ac.uk 


\title{
Discounted Stochastic Games with No Stationary Nash Equilibrium: Two Examples
}

\author{
Yehuda Levy
}

March 27, 2013 


\section{DISCOUNÆED STOCHASTIC GAMES WITH NO STATIONARY NASH \\ 2 EQUILIBRIUM: TWO EXAMPLES \\ 3 \\ 4 \\ YeHudA LEVY*}

We present two examples of discounted stochastic games, each with a continuum of states, finitely many players and actions, that possess no stationary equilibria. The first example has deterministic transitions - an assumption undertaken in most of the early applications of dynamics games in economics - and perfect information, and does not possess even stationary approximate equilibria or Markovian equilibria. The second example satisfies, in addition to stronger regularity assumptions, that all transitions are absolutely continuous with respect to a fixed measure - an assumption that has been widely used in more recent economic applications. This assumption has been undertaken in several positive results on tha existence of stationary equilibria in special cases, and in particular guarantees 1 the existence of stationary approximate equilibria.

KeYworaDS: Stochastic Game, Discounting, Stationary Equilibrium.

15 16

\section{INTRODUCTION}

The question of the existence of stationary equilibria in discounted stochastic games witł uncountable state spaces has received much attention. The purpose of thios paper is to show that such games need not possess equilibria in stationaryzstrategies, neither in the framework of deterministic transitions - used in many of the early applications of dynamics games in economics nor in the more restricted - but much studied in recent years - setting of

Center for take Study of Rationality, and Department of Mathematics, The Hebrew University of Jęusalem, 91904 Jerusalem, Israel.

Tel.: (972-2)-65254135, Fax: (972-2)-6513681, E-mail: john.calculus@gmail.com

${ }^{*}$ Research supported in part by Israel Science Foundation grant 1596/10.

I am grateful t36 Abraham Neyman for going over the drafts of this paper and providing countless correetions and suggestions. I thank Eilon Solan for pointing out the work [44]

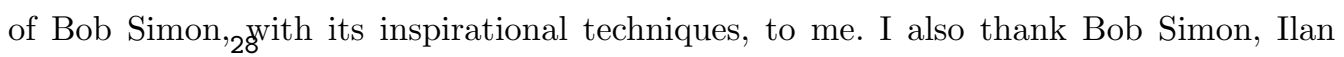
Nehama, and Ziv Hellman for many helpful conversations, and the anonymous referees who performed extremely diligent proofreadings. 
absolutely continuous continuous transitions, even when the action sets are finite (and state-invariant), and the player set is finite.

The increasing usefulness of stochastic games in modeling economic situations, combined with the simplicity and universality of stationary strategies, has made equilibrium existence and characterization results a very active area of research. However, it had been unknown whether the general models of such games did indeed possess stationary equilibria, which were known to exist in the case of discrete state spaces.

Stochastic games were introduced by Shapley (1953). In a stochastic game, players play in discrete stages, with stochastic transitions between states chosen using distributions determined by the state and action. In the $\beta$-discounted game, each player receives the $\beta$-discounted sum of the stream of his stage-by-stage payoffs. A particular class of strategies, the stationary strategies, in which a player makes his decision based only on the current state, has been particularly studied in games with discounted payoffs.

There are two main reasons for this focus. First of all, stationary strategies are the natural class of strategies for the discounted payoff evaluation, as sub-games that are defined by different histories but begin at the same state are strategically equivalent: players will have the same preferences over plays in one sub-game as in the other. The view that strategies should only depend on payoff-relevant data in the discounted game is highlighted in [27], where it motivates the development of the concept of Markov Perfect Equilibria. In [18] this view is called the subgame-consistency principle, which is described succinctly in [19] as "the behaviour principle according to which a player's behaviour in strategically equivalent subgames should be the same, regardless of the different paths by which these subgames might be reached." The other main reason for focusing on the class of stationary strategies is because of their simplicity and easy implementation; to quote [15], "An equilibrium which does not display minimal regularity through 
time - maybe stationarity - is unlikely to generate the coordination between agents that it assumes."

Results for existence of equilibrium in stationary strategies have appeared in increasing generality: [41] for zero-sum games with finite state spaces; [26] for zero-sum games with general state spaces; [13, 45, 38, 42] for nonzero-sum game with finite state space; [35] for non-zero-sum games with countable state space. [43] presented an argument for the non-zero-sum game with general state space, but the proof is flawed. ${ }^{1}$ (It is also worth noting that existence of equilibria in general (i.e., behavioral) strategies was established $^{2}$ in [28].) A survey of these and other results can be found in [11].

Early economic applications of stochastic games (e.g., [22, 29, 39, 6, 37]) used models with deterministic transitions, with transitions representing changes in accumulated resource, wealth, consumer percentages, etc. However, as existence results for stationary equilibria in general classes proved to be elusive, it became common to assume additional continuity conditions on the transitions; in particular, many works have undertaken the assumption which we term the absolute continuity condition, henceforth ACC, which stipulates that all transition measures are absolutely continuous w.r.t. some fixed measure on the state space. This and similar assumptions have been also proven to be natural in some economic settings, e.g., $[2,9,32,33]$ and in particular [10], where this assumption is justified by the presence of 'noise' in the transitions. ${ }^{3}$ (A survey of applications of stochastic games in economics can be found in [3].) This assumption adds to the structure of the

\footnotetext{
${ }^{1}$ This was pointed out in [12].

${ }^{2}$ An alternative proof, under the absolute continuity discussed below, was given in [40]; see also [25].

${ }^{3}$ In [10], the state space has a specific structure: a product structure. The state is chosen then by a compound process of choosing the first coordinate and then, conditional on that choice, the second coordinate via a distribution which is absolutely continuous.
} 
game, and allows for the use of tools that cannot be applied in the general setup. Indeed, under ACC, it has been shown:

(I) There exists stationary $\varepsilon$-equilibria, [31].

(II) There exists stationary extensive-form correlated equilibria, [34]. (Similar results were provided in [9] and [17].)

The purpose of this paper is to present two examples which give negative answers to the question of existence of stationary equilibria in the deterministic model and in the ACC model, respectively.

The first example is of a discounted stochastic games with uncountable state space and deterministic transitions and that does not possess $\varepsilon$-equilibria in stationary strategies. In addition, it satisfies other properties that contrast with other results in the literature. Specifically, for each discount factor $\beta>0$, we construct a game with the following properties:

(1) For $\varepsilon>0$ small enough, stationary $\varepsilon$-equilibria do not exist.

(2) Stationary extensive-form correlated equilibria do not exist.

(3) The game has finite action spaces, perfect information, ${ }^{4}$ and deterministic transitions.

(4) For $\varepsilon>0$ small enough, if payoffs, transitions, ${ }^{5}$ and discount factor are perturbed less than $\varepsilon$ the resulting game still does not possess stationary $\varepsilon$-equilibria. (A formal statement of this robustness appears at the beginning of Section 3.)

(5) The game does not possess sub-game perfect Markovian equilibria. ${ }^{6}$

(6) For any $\varepsilon>0$, there is a perturbation of our example of less than $\varepsilon$ which does not possess Markovian equilibria. ${ }^{7}$

\footnotetext{
${ }^{4}$ In each state, there is only one player whose action has an affect on the transitions or the stage payoffs.

${ }^{5}$ In the total variation norm.

${ }^{6}$ Markovian strategies are those which allow players to condition their choice of mixed action both on the current state and the number of stages played so far; see Section 3.6.

${ }^{7}$ Markovian equilibria which are not sub-game perfect do exist in the unperturbed
} 
(7) The state space is compact, the payoff function is continuous, and the transitions are weakly continuous.

We remark that property (2) follows immediately from properties (1) and (3), while property (4) follows from property (1); see Section 3.5. We further note that the properties (I) and (II), listed above to hold in the ACC model, contrast with properties (1) and (2) of our first example, and therefore show that the study of the general model of stochastic games (and, in particular, of deterministic games) is very different than that of the ACC model.

Hence, we are prompted to give a second example, which does satisfy the ACC assumption. This example has the following stronger properties:

(1) Stationary equilibrium does not exist for any discount factor. (This contrasts the first example, in which the construction depends on the discount factor.)

(2) The state space is compact, payoffs are continuous, and transitions are norm-continuous.

The construction of this example, however, is somewhat more delicate. It relies on certain anomalies in the manifold of Nash equilibria for normalform games. In particular, we take advantage of the existence of a twoperson game whose set of equilibrium is homeomorphic to a circle (thus connected but not simply connected) and each equilibrium of it is stable in the appropriate sense; see, [21, pp. 1034].

We note that since the action spaces are finite in both examples, the transitions are trivially norm-continuous (also termed strongly continuous) in the actions. This contrasts an example given in [17] of a two-stage extensiveform game without an equilibrium. As [10] mentions, in representing this example as a stochastic game, one has to allow for transitions that are not strongly continuous on the infinite action spaces. Therefore, the example from [17] does not fit into the models that are usually studied in works version of the example. 
establishing equilibrium existence results.

Finally, we remark that our second example, which satisfies ACC, is easily seen not to be robust to perturbations, and can be shown to possess Markovian equilibria. It is not known if these are implications of the ACC condition $^{8}$ or if counter-examples satisfying ACC but which either do not possess Markovian equilibria or are robust to perturbations can be found.

In Section 2 we present the formal stochastic game model. The examples are presented in Sections 3 and 4, with some technical proofs in the latter section left for the appendix. Sections 3 and 4 both begin with layouts of their contents.

\section{STOCHASTIC GAME MODEL}

The components of a discounted stochastic game with a continuum of states and finitely ${ }^{9}$ many actions are the following:

- A standard Bore ${ }^{10}$ space $\Omega$ of states.

- A finite set $\mathcal{P}$ of players.

- A finite set of actions $I^{p}$ for each $p \in \mathcal{P}$. Denote $\bar{I}=\prod_{p \in \mathcal{P}} I^{p}$

- A discount factor $\beta \in(0,1)$.

- A bounded payoff function $r: \Omega \times \bar{I} \rightarrow \mathbb{R}^{\mathcal{P}}$, which is Borel-measurable.

- A transition function $q: \Omega \times \bar{I} \rightarrow \Delta(\Omega)$, which is Borel-measurable. ${ }^{11}$

The game is played in discrete time. If $z \in \Omega$ is a state at some stage of the game and the players select an action profile $a \in \bar{I}$, then $q(z, a)$ is the conditional (given the past) probability distribution of the next state of

\footnotetext{
${ }^{8}$ Under ACC, incorrect proofs of the existence of subgame perfect Markovian equilibria have appeared in [8] and [1].

${ }^{9}$ This is a particular case of the general model, which allows for compact actions spaces that are state-dependent; see, e.g., [28].

${ }^{10}$ That is, a space that is homeomorphic to a Borel subset of a complete, metrizable space.

${ }^{11}$ Where $\Delta(\Omega)$, the space of regular Borel probability measures on $\Omega$, possesses the
} Borel structure induced from the topology of narrow convergence. 
the game. A stationary strategy for Player $p$ is a behavioral strategy that depends only on the current state; equivalently, it is a Borel-measurable ${ }^{12}$ mapping that associates with each state $z \in \Omega$ a probability distribution on the set $I^{p}$.

For any profile of behavioral strategies $\sigma=\left(\sigma^{p}\right)_{p \in \mathcal{P}}$ of the players and every initial state $z_{1}=z \in \Omega$, a probability measure $P_{z}^{\sigma}$ and a stochastic process $\left(z_{n}, a_{n}\right)_{n \in \mathbb{N}}$ are defined on $H^{\infty}:=(\Omega \times \bar{I})^{\mathbb{N}}$ in a canonical way, where the random variables $z_{n}, a_{n}$ describe the state and the action profile chosen by the players, respectively, in the $n$-th stage of the game (see, e.g., [7]). The expected payoff vector under $\sigma$, in the game starting from state $z$, is:

$$
\gamma_{\sigma}(z)=E_{z}^{\sigma}\left(\sum_{n=1}^{\infty} \beta^{n-1} r\left(z_{n}, a_{n}\right)\right) .
$$

Let $\Sigma^{p}$ denote the set of behavioral strategies for Player $p \in \mathcal{P}$, and $\Sigma=$ $\prod_{p \in \mathcal{P}} \Sigma^{p}$. A profile $\sigma \in \Sigma$ will be called a Nash equilibrium if

$$
\gamma_{\sigma}^{p}(z) \geq \gamma_{\left(\tau, \sigma^{-p}\right)}^{p}(z), \forall p \in \mathcal{P}, \forall z \in \Omega, \forall \tau \in \Sigma^{p}
$$

and it will be called an $\varepsilon$-Nash equilibrium if

$$
\gamma_{\sigma}^{p}(z) \geq \gamma_{\left(\tau, \sigma^{-p}\right)}^{p}(z)-\varepsilon, \forall p \in \mathcal{P}, \forall z \in \Omega, \forall \tau \in \Sigma^{p}
$$

Denote, for every stationary $\sigma \in \Sigma$, every $z \in \Omega$, and every $a \in \prod_{p \in \mathcal{P}} \Delta\left(I^{P}\right)$,

$$
X_{\sigma}(z, a):=r(z, a)+\beta \int_{\Omega} \gamma_{\sigma}(t) d q(z, a)(t)
$$

By way of iterations, one can show that for stationary $\sigma \in \Sigma$,

$$
\gamma_{\sigma}(z)=X_{\sigma}(z, \sigma(z))
$$

${ }^{12}$ The measurability is required so that the payoffs in the game be well-defined. In certain classes of games, e.g., those with purely atomic transitions, this assumption can be relaxed, at a cost of the constructibility of the strategies. For more on this matter, see the discussion in [23]. 
For stationary $\sigma \in \Sigma$, it is easily shown that (2.2) implies ${ }^{13}$ that

$$
X_{\sigma}^{p}(z, \sigma(z)) \geq X_{\sigma}^{p}\left(z,\left(b, \sigma^{-p}(z)\right)\right), \forall p \in \mathcal{P}, \forall z \in \Omega, b \in I^{p}
$$

i.e., that for all $z, \sigma(z)$ is an equilibrium in the game with payoff $X_{\sigma}(z, \cdot)$, and that (2.3) implies ${ }^{14}$ that

$$
X_{\sigma}^{p}(z, \sigma(z)) \geq X_{\sigma}^{p}\left(z,\left(b, \sigma^{-p}(z)\right)\right)-\varepsilon, \forall p \in \mathcal{P}, \forall z \in \Omega, \forall b \in I^{p}
$$

i.e., that for all $z, \sigma(z)$ is an $\varepsilon$-Nash equilibrium in the game $X_{\sigma}(z, \cdot)$.

Definition 2.0.1 A stochastic game is said to satisfy the Absolute Continuity Condition (ACC) if there is $\nu \in \Delta(\Omega)$ such that for all $z \in \Omega, a \in \bar{I}$, $q(z, a)$ is absolutely continuous w.r.t. $\nu$.

REMARK 2.0.2 One might think to relax the definition of Nash equilibrium in stationary strategies in games satisfying ACC by requiring that (2.2) only hold for $\nu$-a.e. $z \in \Omega$. However, [36] shows that existence of this weaker equilibrium concept would imply existence of the stronger concept, via a simple modification of the "a.e.-equilibrium" on a $\nu$-null set.

We also mention two standard notations we will use; others will be introduced as needed:

- For a bounded real-valued function $f,\|f\|_{\infty}=\sup |f|$, where the supremum is taken over the entire domain of $f$.

- If $p$ is a mixed action over an action space $A$ and $a \in A$, then $p[a]$ denotes the probability that $p$ chooses $a$.

\footnotetext{
${ }^{13}$ In fact, they are equivalent; both directions follow from standard dynamic programming results.

${ }^{14}$ In the converse, (2.7) implies that $\sigma$ is an $\frac{\varepsilon}{1-\beta}$-equilibrium.
} 
In this section, we construct, for any given $\beta \in(0,1)$, a stochastic game $\left(\Omega, \mathcal{P},\left(I^{p}\right), \beta, r, q\right)$, with deterministic transitions and perfect information, that does not possess a stationary (measurable ${ }^{15}$ ) equilibrium. In fact, we will deduce a stronger result for this game: There exists $\varepsilon>0$ such that if $r^{\prime}: \Omega \times \bar{I} \rightarrow \mathbb{R}^{\mathcal{P}}$ and $q^{\prime}: \Omega \times \bar{I} \rightarrow \Delta(\Omega)$ satisfy the measurability conditions given in the model of Section 2 , and also satisfies $\left|\beta^{\prime}-\beta\right|<\varepsilon$ and $^{16}$

$$
\left\|r^{\prime}(z, a)-r(z, a)\right\|_{\infty}<\varepsilon,\left\|q^{\prime}(z, a)-q(z, a)\right\|<\varepsilon, \forall z \in \Omega, \forall a \in \bar{I}
$$

then the game $\left(\Omega, \mathcal{P},\left(I^{p}\right), \beta^{\prime}, r^{\prime}, q^{\prime}\right)$ does not possess a stationary $\varepsilon$-equilibrium.

Henceforth, let $\beta \in(0,1)$ be a fixed discount factor, let $Y=\{-1,1\}^{\omega}$, where $\omega=\{0,1,2, \ldots\}$, let $T$ denote the left-shift operator on $Y$ defined by $(T x)_{n}=x_{n+1}$, and let $\mu$ denote the Lebesgue measure on $Y$.

Section 3.1 begins with an informal description of the construction. Section 3.2 constructs the example, and Section 3.3 presents some properties of any approximate equilibria in it. Section 3.4 proves that no (measurable) stationary equilibria exist in the unperturbed game, and Section 3.5 deals with the perturbed games. Section 3.6 recalls the definition and properties of Markovian strategies, and Section 3.7 shows how the arguments of Sections 3.3 and 3.4 can be modified to show that equilibria need not exist in Markovian strategies. An elaboration of Section 3.7, as well as a discussion on existence (and elimination) of non-measurable equilibria, can be found in [23].

\footnotetext{
${ }^{15}$ The state space will be a finite product of Cantor sets (plus an isolated point), and the measurability we refer to is with respect to the Lebesgue $\sigma$-algebra. Hence, although we defined strategies to be Borel-measurable, we show an even stronger nonexistence result.
}

${ }^{16}$ The latter distance is the total variation norm. 


\subsection{An Informal Description of the Construction}

We begin this description by allowing a countable set of players - one player in each generation $n \in \omega$. The state space will be $\omega \times Y$, along with a "quitting state" $\overline{0}$; all payoffs are zero after the first transition to the quitting state. The transition from a state $(n, y)$ will either be to state $(n+1, T(y))$ or to $\overline{0}$. In a state $(n, *)$, only Player $n$ 's action has an effect on either payoffs or transitions; we can think of him as the only "active" player. Player $n$ receives payoffs both when he is active, in state $(n, y)$, and in the following state, $(n+1, T(y))$ (if the game has not quit). ${ }^{17}$

Each player can play either $L$ or $R$. The component of the state that affects the structure of the payoff and transition in state $(n, y)$ is the 0 -th bit of $y$, denoted $\kappa(y)$. The key is that we define the payoff and transitions such that if Player $n+1$ would play one particular action with high probability in state $(n+1, T(y))$, then Player $n$ in state $(n, y)$ will want to match Player $n+1$ 's expected action if $\kappa(y)=1$, and will want to mismatch it if $\kappa(y)=-1$. Furthermore, we design the game such that regardless of the mixed action Player $n+1$ 's plans to play in state $(n+1, T(y))$, at least one of the agents that represent Player $n$ in the two possible states preceding $(n+1, T(y))$ will not be indifferent between his own actions.

The modification to finitely many players is done simply: we just have the generations repeat themselves periodically, with some period $M$; the state space becomes $(\{0, \ldots, M-1\} \times Y) \cup\{\overline{0}\}$, with the generation-counter being cyclic. If $M$ is chosen large enough - it will depend on the discount factor - each player will make a decision based only on the payoffs of the current

\footnotetext{
${ }^{17}$ This is reminiscent of models of overlapping generation games: each player can be imagined as being alive for two generations. In the first generation, he is "young" and takes an action, and receives some resulting payoff. In the second generation, he is "old"; he does not take an action but he does receive a payoff as a result of the "young" player's action.
} 
stage and next stage when he is called to play; the payoffs from his next "reincarnation", $M$ stages later, will be negligible and will not affect his decision.

\subsection{Construction}

Fix $\delta<\frac{1}{40}, \varepsilon<\frac{\delta}{20}$, and $M \in \mathbb{N}, M>1$, such that $\sum_{j=M}^{\infty} \beta^{j-1}<\delta$. If $p$ is a mixed action over an action space $A$ and $a \in A$, then $p[a]$ denotes the probability that $p$ chooses $a \in A$.

We will construct the game $\left(\Omega, \mathcal{P},\left(I^{p}\right), \beta, r, q\right)$. Denote $Z=\omega_{M} \times Y$, where $\omega_{M}=\{0, \ldots, M-1\}$. The state space will be $\Omega=Z \cup\{\overline{0}\}$, where $\overline{0}$ is an absorbing $^{18}$ state with payoff 0 for all players.

The set of players in the game will be $\mathcal{P}=\omega_{M}$. Each player's action set is $I=\{L, R\}$. For $n \in \omega_{M}$, let $n^{ \pm}=(n \pm 1)_{\bmod M} \in \omega_{M}$, and define $S: Z \rightarrow Z$ by $S(n, y)=\left(n^{+}, T(y)\right)$. Also for $z=(n, y) \in Z$, we denote:

$$
\kappa(z)=y_{0}, \quad n(z)=n, \quad n^{ \pm}(z)=n^{ \pm}
$$

where $y_{0}$ is the 0 -th bit of $y$. The game is a game of perfect information: that is, for each ${ }^{19} z \in Z$, there is only one player, $n(z)$, whose action has any effect on payoffs or transitions. Fix a state $z \in Z$ :

- Only $n(z)$ and $n^{-}(z)$ receive non-zero payoffs in state $z$. That is, if $p \notin\left\{n(z), n^{-}(z)\right\}$, then $r^{p}(z, \cdot) \equiv 0$.

- The payoff to players $n(z), n^{-}(z)$, and the next state $z^{\prime}$, are all determined only by the action of Player $n(z)$ and are given by the following rules:

\footnotetext{
${ }^{18} \mathrm{~A}$ state $z \in \Omega$ is called an absorbing state of $q(z \mid z, a)=1$ for all action profiles $a$. ${ }^{19}$ In $\overline{0}$, no player's action has an effect.
} 


If $\kappa(z)=1:$
\begin{tabular}{|c|c|c|}
\hline$a^{n(z)}=$ & $L$ & $R$ \\
\hline$r^{n(z)}(z, a)=$ & 0 & 0.3 \\
\hline$r^{n^{-}(z)}(z, a)=$ & $\frac{1}{\beta}$ & 0 \\
\hline$z^{\prime}=$ & $S(z)$ & $\overline{0}$ \\
\hline
\end{tabular}

If $\kappa(z)=-1:$
\begin{tabular}{|c|c|c|}
\hline$a^{n(z)}=$ & $L$ & $R$ \\
\hline$r^{n(z)}(z, a)=$ & 0.7 & 0 \\
\hline$r^{n^{-}(z)}(z, a)=$ & $\frac{1}{\beta}$ & 0 \\
\hline$z^{\prime}=$ & $\overline{0}$ & $S(z)$ \\
\hline
\end{tabular}

\subsection{Observations and Characterization of Equilibria}

Fix a stationary $\varepsilon$-equilibrium profile $\sigma$ of this game. Recall the notation $\gamma_{\sigma}$ and $X_{\sigma}$ from Section 2. For $p \in \mathcal{P}$ and $z \in Z \subseteq \Omega, \sigma^{p}(z)$ will denote the probability distribution on $\{L, R\}$ induced by Player $p$ 's mixed action in state $z$. Recall the definition of $n(z)$ from (3.1), and denote further that:

$$
\ell(z)=\sigma^{n(z)}(z)[L]
$$

We will study the relationship between $\ell(S(z))$ and $\ell(z)$. Recall that in the game that starts at state $z$, the player that is active in state $z, n(z)$, receives a zero payoff in stages $t=2, \ldots, M$. Therefore,

Therefore, if

$$
\begin{aligned}
\gamma_{\sigma}^{n(z)}(S(z)) & =E_{S(z)}^{\sigma}\left(\sum_{t=1}^{\infty} \beta^{t-1} r^{n(z)}\left(z_{t}, a_{t}\right)\right) \\
& =E_{S(z)}^{\sigma}\left(r^{n(z)}\left(S(z), a_{1}\right)\right)+E_{S(z)}^{\sigma}\left(\sum_{t=M}^{\infty} \beta^{t-1} r^{n(z)}\left(z_{t}, a_{t}\right)\right) .
\end{aligned}
$$

$$
K_{\sigma}(z, \cdot):=r^{n(z)}(z, \cdot)+\beta q(S(z) \mid z, \cdot) r^{n(z)}(S(z), \sigma(S(z)))
$$

the inequality $\beta \sum_{t=M}^{\infty} \beta^{t-1}\|r\|_{\infty}=\sum_{t=M}^{\infty} \beta^{t-1}<\delta$ implies that

$$
\left\|X_{\sigma}^{n(z)}(z, \cdot)-K_{\sigma}(z, \cdot)\right\|_{\infty}<\delta
$$

Notation 3.3.1 Let $\left\langle\alpha_{L} ; \alpha_{R}\right\rangle$, for $\alpha_{L}, \alpha_{R} \in \mathbb{R}$, denote the single-player decision that gives payoff $\alpha_{L}$ (resp. $\left.\alpha_{R}\right)$ if the player plays $L$ (resp. $R$ ). 


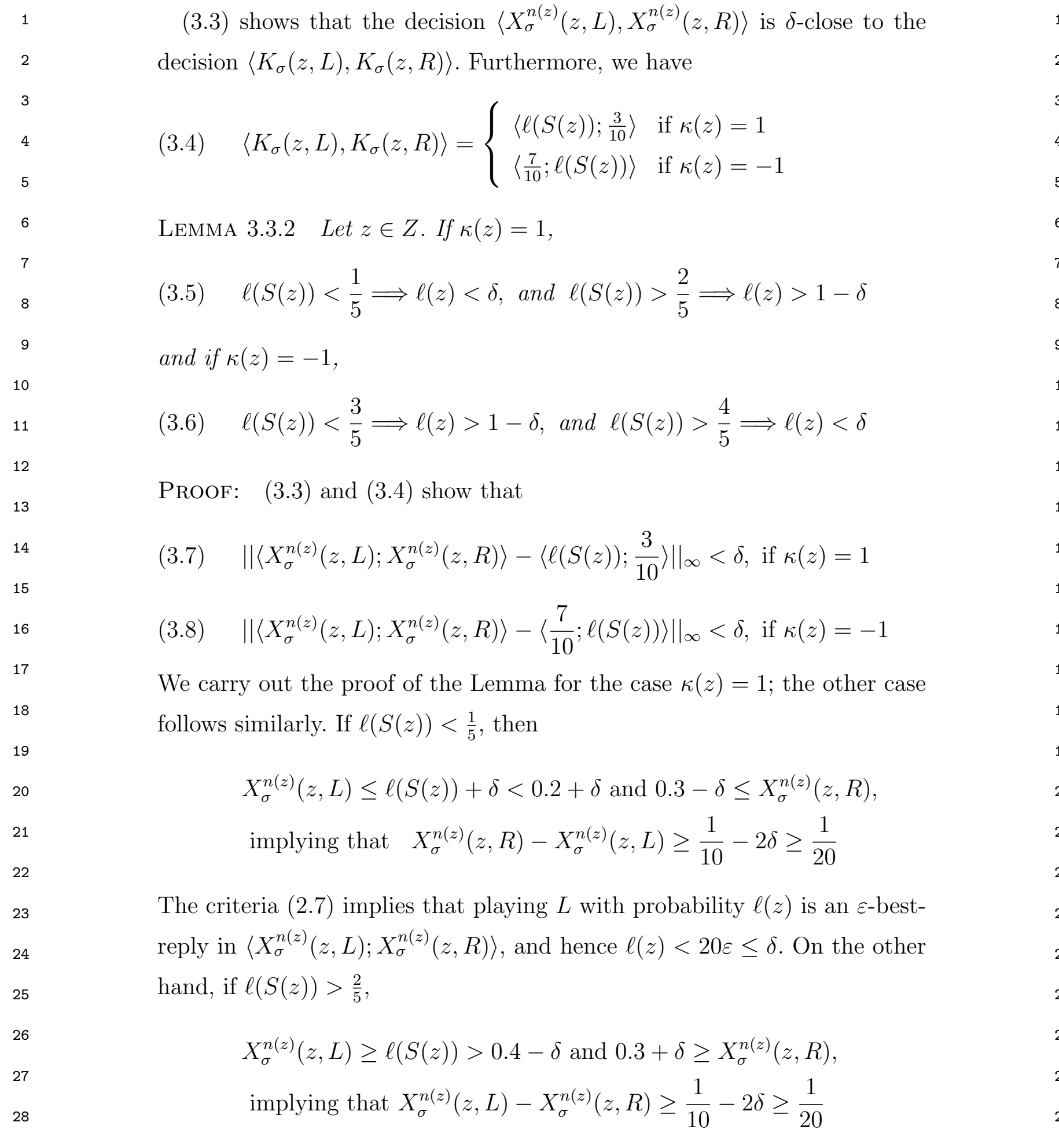

and we similarly derive that in this case, $\ell(z)>1-20 \varepsilon \geq 1-\delta$. Q.E.D. 
14

Definition 3.3.3 A state $z \in Z$ will be called L-quasi-pure (resp. $R$-quasipure) if $\ell(z)>1-\delta$ (resp. $\ell(z)<\delta)$. If $z$ is either $L$-or $R$-quasi-pure, we may simply refer to $z$ as being quasi-pure.

Lemmas 3.3.4 and 3.3.5 contain the properties of $\sigma$ that we will need.

Lemma 3.3.4 If $S(z)$ is quasi-pure in $\sigma$, then so is $z$. If the former is a-quasi-pure $(a \in\{L, R\})$, then the latter is as well if and only if $\kappa(z)=1$.

Proof: The lemma follows by repeated use of Lemma 3.3.2 (we shorten 'quasi-pure' to 'q.p.' here):

- If $S(z)$ is $L$-q.p. and $\kappa(z)=1$, then $\ell(S(z))<\delta<\frac{1}{5}$, so $\ell(z)<\delta$.

- If $S(z)$ is $L$-q.p. and $\kappa(z)=-1$, then $\ell(S(z))<\delta<\frac{3}{5}$, so $\ell(z)>1-\delta$.

- If $S(z)$ is $R$-q.p. and $\kappa(z)=1$, then $\ell(S(z))>1-\delta>\frac{2}{5}$, so $\ell(z)>1-\delta$.

- If $S(z)$ is $R$-q.p. and $\kappa(z)=-1$, then $\ell(S(z))>1-\delta>\frac{4}{5}$, so $\ell(z)<\delta$.

Q.E.D.

Lemma 3.3.5 For any $z \in Z$, at least one of the two states in $S^{-1}(z)$ is quasi-pure. (Note that this is so even if $z$ is not quasi-pure.)

Proof: We must have at least one of the following two inequalities:

$$
\ell(S(z))>\frac{2}{5}, \ell(S(z))<\frac{3}{5}
$$

Suppose that the left inequality holds. Lemma 3.3.2 then shows that if $z^{\prime} \in S^{-1}(z)$ with $\kappa\left(z^{\prime}\right)=1$, then $\ell\left(z^{\prime}\right)>1-\delta$ and hence $z^{\prime}$ is $L$-quasi-pure. In the case of the right inequality, we deduce similarly that if $z^{\prime \prime} \in S^{-1}(z)$ with $\kappa\left(z^{\prime \prime}\right)=-1$, then $z^{\prime \prime}$ is also $L$-quasi-pure.

Q.E.D.

REMARK 3.3.6 It's easy to describe a (pure) equilibrium in behavioral strategies: The player who begins the game plays, say, $L$. Thereafter, as 
long as the quitting state $\overline{0}$ is not reached, each player will match (resp.

1 mismatch) the action of the player before him if preceding state $z$ satisfies $\kappa(z)=1$ (resp. $\kappa(z)=-1$ ). Indeed, [28] guarantees the existence of equilibria in behavioral strategies. In fact, the proof there in fact shows that in perfect information games, pure behavioral equilibria exist; this had also been demonstrated earlier in [16].

\subsection{Nonexistence of Stationary Equilibria: The Unperturbed Game}

Recall that $\mu$ is the Lebesgue-measure on $Y$, and let $\lambda$ be the uniform measure on $\omega_{M}$; let $\nu=\lambda \times \mu$. Assume that $\sigma$ is a stationary $\varepsilon$-equilibrium, as in Section 3.3, measurable w.r.t. $\nu$. We shall use Lemmas 3.3.4 and 3.3.5 to show that $\sigma$ cannot be a $\left(\nu\right.$-measurable $\left.{ }^{20}\right)$ equilibrium. Assume, to the contrary, that it is.

Lemma 3.4.1 Let $\Xi=\{z \in Z \mid z$ is not quasi-pure $\}$. Then $\nu(\Xi)=0$.

Proof: By assumption, $\Xi$ is $\nu$-measurable. Lemma 3.3.4 implies that

$$
S(\Xi) \subseteq \Xi
$$

Let $\iota: Z \rightarrow Z$ be the involution defined such that $\iota(n, y)$ is obtained from $(n, y)$ by changing only the 0 -th bit of $y$. Lemma 3.3.5 then implies that

\footnotetext{
${ }^{20}$ Where $\nu$ is also a measure on $\Omega$ via inclusion.
} 
$S$ and $\iota$ are both $\nu$-preserving. ${ }^{21}$ Also observe that $S(\Xi)$ is $\nu$-measurable. ${ }^{22}$

$$
2 \nu(\Xi)=\nu(\Xi)+\nu(\iota(\Xi))=\nu\left(S^{-1}(S(\Xi))\right)=\nu(S(\Xi)) \leq \nu(\Xi)
$$

Hence, $\nu(\Xi)=0$.

Q.E.D.

Define the map $g: Z \rightarrow\{-1,1\}$ by $g(z)=1$ if and only if $z$ is $L$-quasipure. Denote for all $y, y^{\prime} \in Y, D\left(y, y^{\prime}\right)=\left\{j \in \omega \mid y_{j} \neq y_{j}^{\prime}\right\}$, and if $D\left(y, y^{\prime}\right)$ is finite, $N\left(y, y^{\prime}\right)=\# D\left(y, y^{\prime}\right), M\left(y, y^{\prime}\right)=\max D\left(y, y^{\prime}\right)$.

LEMma 3.4.2 For each $n \in \omega_{M}$, $\mu$-almost every $y \in Y$, we have

$$
g(n, y)=(-1)^{N\left(y, y^{\prime}\right)} g\left(n, y^{\prime}\right), \forall y^{\prime} \in Y \text { s.t. } N\left(y, y^{\prime}\right)<\infty
$$

Proof: By Lemma 3.3.4 and Lemma 3.4.1, we see that for almost every $z=(n, y) \in Z, g(z)=y_{0} \cdot g(S(z))$, and hence for all $k$ and a.e. $z$,

$$
g(z)=y_{0} \cdots \cdot y_{k-1} \cdot g\left(S^{k}(z)\right)
$$

If $N\left(y, y^{\prime}\right)<\infty, z=(n, y), z^{\prime}=\left(n, y^{\prime}\right)$, then $S^{M\left(y, y^{\prime}\right)}(z)=S^{M\left(y, y^{\prime}\right)}\left(z^{\prime}\right)$ and $(-1)^{N\left(y, y^{\prime}\right)}=\prod_{j \leq M\left(y, y^{\prime}\right)} \frac{y_{j}}{y_{j}^{\prime}}$; hence the result follows. Q.E.D.

Proposition 3.4.3 There does not exist a $\mu$-measurable function $f: Y \rightarrow$ $\{-1,1\}$, such that for a.e. $y \in Y$,

$$
f(y)=(-1)^{N\left(y, y^{\prime}\right)} f\left(y^{\prime}\right), \forall y^{\prime} \in Y \text { s.t. } N\left(y, y^{\prime}\right)<\infty
$$

${ }^{21}$ Recall that a mapping $\psi$ on a measure space $(\Omega, \lambda)$ is measure-preserving if $\lambda\left(\psi^{-1}(A)\right)=\lambda(A)$ for all $\lambda$-measurable $A \subseteq \Omega$. ८ is clearly $\nu$-preserving, and the map $n \rightarrow n^{+}$in $\omega_{M}$ is clearly $\lambda$-preserving; that shifts are Lebesgue-measure preserving, and that the product of measure-preserving systems are also measure-preserving, are standard results in ergodic theory.

${ }^{22}$ This is easy to establish in the case that $\Xi \subseteq\{1\} \times Y$ or $\Xi \subseteq\{-1\} \times Y$, and the general case follows. 
Proposition 3.4.3 contradicts 3.4.2, and therefore completes our proof

1 that there are no stationary equilibria. Before the proof, we recall several notions: Let $S_{\omega}$ denote the set of permutations $\pi$ on $\omega$ such that $\exists N \in$ $\omega, \forall n>N, \pi(n)=n$. $S_{\omega}$ acts on $Y$ by $(\pi(y))_{n}=y_{\pi^{-1}(n)}$. A transposition (on $\omega$ ) is an element $\pi$ of $S_{\omega}$ for which there are $i, j \in \omega$ with $\pi(i)=j$ and $\pi(j)=i$, and $\pi(k)=k$ for all $k \neq i, j$. It is well known that every element of $S_{\omega}$ is a composition of finitely many transpositions. We also denote by $\chi_{j}: Y \rightarrow Y$ the involution which changes only the $j$-th bit of the sequence. Proof: Suppose that we did have such an $f$. Denote

$$
L=\{y \in Y \mid f(y)=1\}
$$

Note that $\mu(L)=\frac{1}{2}$ : first, note that $f(y)=-f\left(\chi_{0}(y)\right)$ for $\mu$-a.e. $y$. Hence, for a.e. $y$, exactly one of the following options holds: $y \in L$ or $\chi_{0}(y) \in L$ (equivalently, $\left.y \in \chi_{0}(L)\right)$. Hence $\mu\left(\chi_{0}(L) \cap L\right)=0, \mu\left(\chi_{0}(L) \cup L\right)=1$.

On the other hand, let $\pi \in S_{\omega}$ and $y \in Y$ for which (3.13) holds. We contend that $y \in L$ if and only if $\pi(y) \in L$; it's enough to check this in the case that $\pi$ is a transposition. We have either $\pi(y)=y$ or $\pi(y)=\chi_{i} \circ \chi_{j}(y)$, so $N(\pi(y), y) \in\{0,2\}$. Therefore, $\mu(\pi(L) \Delta L)=0$ for all $\pi \in S_{\omega}$, where $\Delta$ denotes the symmetric difference of sets. By the Hewitt-Savage zero-one law, $\mu(L)=0$ or $\mu(L)=1$, a contradiction.

Q.E.D.

\subsection{Nonexistence of Stationary Equilibria: The Perturbed Games}

The following lemma can be established along standard lines:

Proposition 3.5.1 Let $\Gamma=\left(\Omega, \mathcal{P},\left(I^{p}\right), \beta, r, q\right)$ be a stochastic game and $\epsilon>0$. Then there is $\eta>0$ such that if $\Gamma^{\prime}=\left(\Omega, \mathcal{P},\left(I^{p}\right), \beta^{\prime}, r^{\prime}, q^{\prime}\right)$ is another game with the same state / player / action spaces, such that $\left|\beta^{\prime}-\beta\right|<\eta$,

$$
\left\|r^{\prime}(z, a)-r(z, a)\right\|_{\infty}<\eta,\left\|q^{\prime}(z, a)-q(z, a)\right\|<\eta, \forall z \in \Omega, \forall a \in \bar{I}
$$


then for any behavioral strategy profile $\sigma$, letting $\gamma_{\sigma}(z), \gamma_{\sigma}^{\prime}(z)$ denote the expected payoffs in $\Gamma, \Gamma^{\prime}$ starting with state $z$, we have $\left\|\gamma_{\sigma}-\gamma_{\sigma}^{\prime}\right\|_{\infty}<\epsilon$.

It therefore follows that stationary $\epsilon$-equilibria in $\Gamma^{\prime}$ are stationary $3 \epsilon$ equilibria in $\Gamma$, a contradiction if $3 \epsilon$ is small enough.

\subsection{Markovian Strategies: The Concept and Dynamic Programming}

A Markovian strategy is a behavioral strategy in which a player's action can depend on the current stage of the game ${ }^{23}$ and the current state. A Markovian strategy $\sigma^{p}$ for a player $p \in \mathcal{P}$ is given by a sequence, $\sigma^{p}=$ $\left(\sigma_{1}^{p}, \sigma_{2}^{p}, \ldots\right)$, where for each $m \in \mathbb{N}, \sigma_{m}^{p}$ is a measurable map $\Omega \rightarrow \Delta\left(I^{p}\right)$. We will show that our example does not possess subgame perfect Markovian equilibria. ${ }^{24}$ Afterwards, we will show that there are arbitrarily small perturbations of our example that do not possess Markovian equilibria. ${ }^{25}$

We adopt the various notations of Section 2. Furthermore, if $\sigma=\left(\sigma_{1}, \sigma_{2}, \ldots\right)$ is a Markovian strategy profile, let $\sigma_{* m}$ be the Markovian strategy profile $\left(\sigma_{m+1}, \sigma_{m+2}, \ldots\right)$, and we generalize the notation of Section 2 by defining for each state $z \in \Omega$, and for a mixed action profile $a \in \prod_{p \in \mathcal{P}} \Delta\left(I^{P}\right)$,

$$
X_{\sigma_{* m}}^{p}(z, a):=r(z, a)+\beta \int_{\Omega} \gamma_{\sigma_{* m}}(t) d q(z, a)(t)
$$

Proposition 3.6.1 A Markovian strategy profile $\sigma$ is a subgame perfect equilibrium iff for every state $z \in \Omega$, every $m \in \mathbb{N}$, and every $z \in \Omega$,

$$
X_{\sigma_{* m}}^{p}\left(z, \sigma_{m}(z)\right) \geq X_{\sigma_{* m}}^{p}\left(z,\left(b,\left(\sigma_{m}\right)^{-p}(z)\right)\right), \forall p \in \mathcal{P}, \forall b \in I^{p}
$$

${ }^{23}$ That is, how much time has elapsed since play began.

${ }^{24} \mathrm{~A}$ similar argument can show that our example does not posses a Markovian subgame perfect $\varepsilon$-equilibrium - i.e., a Markovian strategy profile which induces an $\varepsilon$-equilibrium in any subgame - but we will settle for simplicity.

${ }^{25}$ The nonperturbed example possesses Markovian equilibria: for all $p \in \mathcal{P}$, let $\sigma_{1}^{p}(z)=$ $R$ for $\kappa(z)=1$ and $\sigma_{1}^{p}(z)=L$ for $\kappa(z)=-1$, let $\sigma_{2}^{p}(z)=R$ for all $z$, and let $\sigma_{k}^{p}(z)$ be arbitrary for $k \geq 3$.

ectaart.cls ver. 2006/04/11 file: stochastic_game_no_eq.tex date: March 27, 2013 


\subsection{Nonexistence of Markovian Equilibrium in Example I}

Fix some $\beta$. We will show that the game $\Gamma=\left(\Omega, \mathcal{P},\{L, R\}^{\mathcal{P}}, \beta, r, q\right)$ defined in Section 3.2 does not have a subgame perfect Markovian equilibria. At the end of this section we remark how to find pertubations of $\Gamma$ which do not possess Markovian equilibria. Assume, by way of contradiction, a fixed measurable subgame perfect Markovian equilibrium profile $\sigma$.

Definition 3.7.1 For each $m \in \mathbb{N}$, denote $\ell_{m}(z)=\sigma_{m}^{n(z)}(z)[L]$. A state $z \in Z$ will be called $(L, m)$-pure (resp. $(R, m)$-pure) if $\ell_{m}(z)=1$ (resp. $\left.\ell_{m}(z)=0\right)$. If $z$ is either $(L, m)$ - or $(R, m)$-pure, we may simply refer to $z$ as being m-pure.

The following lemma parallels Lemmas 3.3.4 and 3.3.5, and can be deduced along similar lines:

LEMma 3.7.2 If $S(z)$ is $m+1$-pure, then $z$ is m-pure. If the former is $(a, m+1)$-pure $(a \in\{L, R\})$, then the latter is $(a, m)$-pure if and only if $\kappa(z)=1$. Furthermore, for any $z \in Z, m \in \mathbb{N}$, at least one of the two states in $S^{-1}(z)$ is m-pure.

Lemma 3.7.3 For each $m \in \mathbb{N}$, let $\Xi_{m}$ denote the set of states which are not $m$-pure. Then $\nu\left(\Xi_{m}\right)=0$ for all $m \in \mathbb{N}$.

Proof: As in the proof of Lemma 3.4.1, we show that, $2 \nu\left(\Xi_{m}\right)=\nu\left(S\left(\Xi_{m}\right)\right) \leq$ $\nu\left(\Xi_{m+1}\right)$. Inductively, we see that $2^{k} \cdot \nu\left(\Xi_{m}\right) \leq \nu\left(\Xi_{m+k}\right)$, and in particular $2^{k} \cdot \nu\left(\Xi_{m}\right) \leq 1$, for all $k, m \in \mathbb{N}$. Hence $\nu\left(\Xi_{m}\right)=0$.

Q.E.D.

Now, define the map $g: Z \rightarrow\{-1,1\}$ by $g(z)=1$ if and only if $z$ is $(L, 1)$-pure. Lemma 3.4.2 holds for $g$ defined in this manner; by Theorem 3.4.3, such $g$ cannot be measurable, which completes our contradiction. 
Now, let $\Gamma^{\prime}=\left(\Omega, \mathcal{P},\{L, R\}^{\mathcal{P}}, \beta, r, q^{\prime}\right)$ be defined from $\Gamma$ by

1

$q^{\prime}(z, a)=(1-\epsilon) \cdot q(z, a)+\epsilon \cdot \delta_{S(z)}$,

where $\epsilon>0$ and $\delta_{c}$ denotes the Dirac measure at $c$. Its easy to see that every Markovian equilibrium in $\Gamma^{\prime}$ is subgame-perfect, and arguments similar to the ones above show that if $\epsilon$ is small enough then $\Gamma^{\prime}$ does not have a subgame-perfect equilibrium.

\section{EXAMPLE II (WITH ACC)}

In this section, we construct a stochastic game $\left(\Omega, \mathcal{P},\left(I^{p}\right), r, q\right)$ which does not possess a stationary equilibrium for any discount factor $\beta \in(0,1)$. The game has a compact state space, a continuous payoff function, and normcontinuous transitions.

Section 4.1 gives the idea of our construction. Section 4.2 introduces some notation. The construction itself of the fundamental normal-form game takes place in Sections 4.3 and 4.4, modulo a technical claim which is proved in the Appendix. In Section 4.5, the example of a stochastic game without a stationary equilibrium is presented. Section 4.6 discusses what minimal anomalies of the structure of Nash equilibria we take advantage of in our construction. (We remark that Section 4.5 can be read after having only read the description and the properties of the normal-form game provided in Section 4.4; it does not depend directly on Section 4.3.)

\subsection{The Idea of The Construction}

The game we will construct will have state space $[0,1]$, where 1 is an absorbing state with payoff 0 . The payoffs decrease linearly as one moves towards 1 , and the transitions from state $t$ are of two types (or some mixture thereof): uniformly in $[t, 1)$, or quitting to 1 . As such, the game progresses towards the right. 
The transitions will be controlled by a particular pair of players whom we denote $C, D$. These players have no influence over their stage payoff, and each of them influences whether the game is to "continue," i.e., if the transition should be uniform in $[t, 1)$, or is to "quit," i.e., go all the way to the absorbing state. Clearly, then, in state $t<1$, each of these players chooses which way he wishes to influence depending on whether his future average expected payoff in the states to his right is positive or negative.

We seek to build a group of players around $C, D$ with which to implement a mechanism with two main properties in each state $t<1$. The first property is that the action that each of the players $C, D$ plays in response to a future expected positive (resp. negative) payoff in $[t, 1$ ) induces the other players, in any stationary equilibrium, to award that player a negative (resp. positive) stage payoff. From this mechanism (and the particular structure of the game) it will follow that, in any stationary equilibrium, each of the players $C, D$ must always receive a payoff of 0 . However, this contradicts the other main property of the mechanism: the stage payoff to at least one of the players $C, D$ must be non-zero in any stage of play of any stationary equilibrium.

To achieve a mechanism with both these properties, we take advantage of an example presented in [21] in relation to stability properties of equilibria, in which the set of equilibria is homeomorphic to a circle and all equilibria satisfy an appropriate stability property. A particular pair of players, denoted $A, B$, will face a game very $\operatorname{close}^{26}$ to the normal-form game in this example, with small perturbations induced by the actions of the pair $C, D$ (and the resulting best-replies of a team of 'auxiliary' players). As a result, in stationary equilibrium, the action pair played by $A, B$ at any stage will always be near the aforementioned circle of equilibria, but changes in $C, D$ 's action profile (as a function of expected future payoffs) will cause

\footnotetext{
${ }^{26}$ Close in the space of games, treated as a Euclidean space.
} 
$A, B$ 's action pair to move to a different part of the circle, hence inducing both properties of the mechanism that we require.
- Distances in any Euclidean spaces (including spaces of games and spaces of mixed action profiles) are always w.r.t. to the $\|\cdot\|_{\infty}$ norm.

- If $g$ is some payoff vector to some set of players $\mathcal{P}$, and $T \subseteq \mathcal{P}$, then $g^{T}$ denotes the restriction of the vector to the players in $T$.

- If $a$ is an action profile of the players in $\mathcal{P}$, and $T \subseteq \mathcal{P}$, then $a^{T}$ (resp. $a^{-T}$ ) denotes the vector of strategies of players in $T$ (resp. $\mathcal{P} \backslash T$ ).

- If $\Lambda$ is a normal form game on some set of players $\mathcal{P}$, and $\alpha$ is a strategy profile of those players, then $\Lambda(\alpha)$ denotes the resulting payoff vector. If $T \subseteq \mathcal{P}$, then $\Lambda^{T}(\alpha)$ (resp. $\left.\Lambda^{-T}(\alpha)\right)$ denotes the payoff to the players in $T$ (resp. in $P \backslash T$ ).

- For such $\Lambda, \alpha$, and $T \subseteq \mathcal{P}, \Lambda^{T}\left(\cdot, \alpha^{-T}\right)$ denotes the expected normalform game facing the players in $T$ when the other players are restricted to playing $\alpha^{-T}$.

- For a normal-form game $\Lambda, N E(\Lambda)$ is the set of Nash equilibria of $\Lambda$.

- We let $S$ denote the boundary of the square,

$$
S=\{(p, q) \mid-1 \leq p, q \leq 1,(|p|=1) \vee(|q|=1)\} .
$$

We denote the four closed edges of $S$ by $S_{\mathcal{N}}, S_{\mathcal{E}}, S_{\mathcal{S}}, S_{\mathcal{W}}$ for the north, east, south, and west edges, respectively. Note that $S_{\mathcal{N}}=-S_{\mathcal{S}}, S_{\varepsilon}=$ $-S_{\mathcal{W}}$.

- When referring to the set $\{1,-1\}$, for $p \in[0,1],(p, 1-p)$ denotes the probability distribution choosing 1 with probability $p$, and choosing -1 with probability $1-p$.

- Given a set $E$ and a point $x$ in an Euclidean space,

$$
\|x-E\|_{\infty}:=\inf _{y \in E}\|x-y\|_{\infty}
$$




\subsection{Construction from Kohlberg and Mertens' Game}

In this section, we construct for each $\varepsilon>0$ a continuous function $\Gamma_{\varepsilon}$ from the square $S$ to the collection of $3 \times 3$ bimatrix games, i.e., to $\mathbb{R}^{2 \times I \times J}$, where $I=J=\{L, M, R\}$, which will satisfy certain key properties that we discuss below. The motivation for this construction is the following game (we denote the players $A, B$ ), presented by Kohlberg and Mertens (1986), whose set of equilibria is homeomorphic to a circle. ${ }^{27}$

\begin{tabular}{|c|c|c|c|c|}
\hline \multicolumn{4}{|c|}{ The Game $G_{0}$} & Equilibria of $G_{0}$ \\
\hline$A \backslash B$ & $L$ & M & $R$ & \multirow{5}{*}{$\begin{array}{l}(L, L)-\underset{\mid}{\mid}(M, M)-(M, R) \\
\mid(R, L)-(R, M)\end{array}$} \\
\hline$L$ & 1,1 & $0,-1$ & $-1,1$ & \\
\hline$M$ & $-1,0$ & 0,0 & $-1,0$ & \\
\hline$R$ & $1,-1$ & $0,-1$ & $-2,-2$ & \\
\hline \multirow{2}{*}{\multicolumn{4}{|c|}{ Table 4.2.a }} & \\
\hline & & & & Figure 4.2.b \\
\hline
\end{tabular}

Let $E_{1}, \ldots, E_{6}$ denote the 6 pure equilibria, beginning with $(L, L)$ and proceeding clockwise, and let $A_{i}$ denote the closed arc from $E_{i}$ to $E_{i+1, \bmod } 6$ in the space of mixed strategy profiles. The equilibria of $G_{0}$ are precisely the strategies lying on these arcs, i.e., $N E\left(G_{0}\right)=\cup_{j=1}^{6} A_{j}$. For a two-player game $G$, the game $G^{\prime}$, defined by $G^{\prime i}(a, b)=G^{3-i}(b, a)$, is the game where the players and action profiles are switched.

Fix $\varepsilon>0$; we begin by defining mappings $G_{1}, \ldots, G_{6}, G_{Z}:[0,1] \rightarrow$ $\mathbb{R}^{2 \times I \times J}$, and from these we will define $\Gamma_{\varepsilon}$. By construction, for $j=1, \ldots, 6$ and any $t \in[0,1]$, any equilibrium of $G_{j}(t)$ lies along the closed $\operatorname{arc} A_{j} .\left(G_{Z}\right.$, however, has an 'irregularity'.)

\footnotetext{
${ }^{27}$ And is hyperstable in the sense defined there.
} 


$G_{1}(t):=$\begin{tabular}{|c|c|c|c|}
\hline$A \backslash B$ & $L$ & $M$ & $R$ \\
\hline$L$ & $1+\varepsilon, 1+(1-t) \varepsilon$ & $\varepsilon,-1$ & $-1+\varepsilon, 1+t \cdot \varepsilon$ \\
\hline$M$ & $-1,(1-t) \varepsilon$ & 0,0 & $-1, t \cdot \varepsilon$ \\
\hline$R$ & $1,-1$ & $0,-1$ & $-2,-2$ \\
\hline
\end{tabular}

All equilibria in $G_{1}(t)$ lie on the $\operatorname{arc} A_{1}$.

$G_{2}(t):=$\begin{tabular}{|c|c|c|c|}
\hline$A \backslash B$ & $L$ & $M$ & $R$ \\
\hline$L$ & $1+(1-t) \varepsilon, 1$ & $(1-t) \varepsilon,-1$ & $-1+(1-t) \varepsilon, 1+\varepsilon$ \\
\hline$M$ & $-1,0$ & $t \cdot \varepsilon, 0$ & $-1+t \cdot \varepsilon, \varepsilon$ \\
\hline$R$ & $1-t \cdot \varepsilon, 0$ & $-t \cdot \varepsilon,-1$ & $-2,-2$ \\
\hline
\end{tabular}

All equilibria of $G_{2}(t)$ lie along $A_{2}$.

$G_{3}(t):=$\begin{tabular}{|c|c|c|c|}
\hline$A \backslash B$ & $L$ & $M$ & $R$ \\
\hline$L$ & $1,1-2 t \cdot \varepsilon$ & $-t \cdot \varepsilon,-1$ & $-1,1-2\left(t-\frac{1}{2}\right) \varepsilon$ \\
\hline$M$ & $-1,-t \cdot \varepsilon$ & $\varepsilon, t \cdot \varepsilon$ & $-1+\varepsilon,-2\left(t-\frac{1}{2}\right) \varepsilon$ \\
\hline$R$ & $1-\varepsilon,-1$ & $-\varepsilon,-1+t \cdot \varepsilon$ & $-2,-2$ \\
\hline
\end{tabular}

All equilibria of $G_{3}(t)$ lie along $A_{3}$.

For $t<\frac{1}{2}$ or $t>\frac{1}{2}$, the unique equilibrium of $G_{Z}(t)$ is $(M, M)$. More- 
over,

$G_{Z}\left(\frac{1}{2}\right)=$\begin{tabular}{|c|c|c|c|}
\hline$A \backslash B$ & $L$ & $M$ & $R$ \\
\hline$L$ & $1-\varepsilon, 1-\varepsilon$ & $-\varepsilon,-1$ & $-1,1-\varepsilon$ \\
\hline$M$ & $-1,-\varepsilon$ & $\varepsilon, \varepsilon$ & $-1+\varepsilon,-\varepsilon$ \\
\hline$R$ & $1-\varepsilon,-1$ & $-\varepsilon,-1+\varepsilon$ & $-2,-2$ \\
\hline
\end{tabular}

which has pure equilibria $(L, L)$ and $(M, M)$, and the mixed equilibrium,

$$
\left(x^{*}, y^{*}\right)=\left(\left(\frac{2 \varepsilon}{2+\varepsilon}, \frac{2-\varepsilon}{2+\varepsilon}, 0\right),\left(\frac{2 \varepsilon}{2+\varepsilon}, \frac{2-\varepsilon}{2+\varepsilon}, 0\right)\right)
$$

which satisfies

$$
\left\|\left(x^{*}, y^{*}\right)-(M, M)\right\|_{\infty}=\frac{2 \varepsilon}{2+\varepsilon}<\varepsilon
$$

- Since $G_{3}(1)=G_{Z}^{\prime}(1)$, we retrace our steps in the transposed games; we get

$$
G_{5}(t):=G_{2}^{\prime}(1-t)
$$

$$
G_{6}(t):=G_{1}^{\prime}(1-t)
$$

In each of these cases, all equilibria of $G_{j}$ lie along $A_{j}$.

$$
\Gamma_{\varepsilon}(p, q)= \begin{cases}G_{4}\left(\frac{1}{2}(1+p)\right) & \text { if } q=1 \\ G_{5}\left(\frac{1}{2}(1-q)\right) & \text { if } p=1 \\ G_{6}\left(\frac{1}{2}(1-p)\right) & \text { if } q=-1 \\ G_{1}(2(q+1)) & \text { if } p=-1, q \leq-\frac{1}{2} \\ G_{2}\left(2\left(q+\frac{1}{2}\right)\right) & \text { if } p=-1,-\frac{1}{2} \leq q \leq 0 \\ G_{3}(2 q) & \text { if } p=-1,0 \leq q \leq \frac{1}{2} \\ G_{Z}\left(2\left(q-\frac{1}{2}\right)\right) & \text { if } p=-1, \frac{1}{2} \leq q \leq 1\end{cases}
$$


Clearly, $\Gamma_{\varepsilon}$ is well-defined and continuous; one just verifies $G_{1}(1)=G_{2}(0), \ldots$, etc. To better understand $\Gamma_{\varepsilon}$, denote $H_{j}=G_{j}(0)$ for $j=1, \ldots, 6, Z$. Then 2 the map $\Gamma_{\varepsilon}$ is the piecewise linear map given by the following diagram: 3

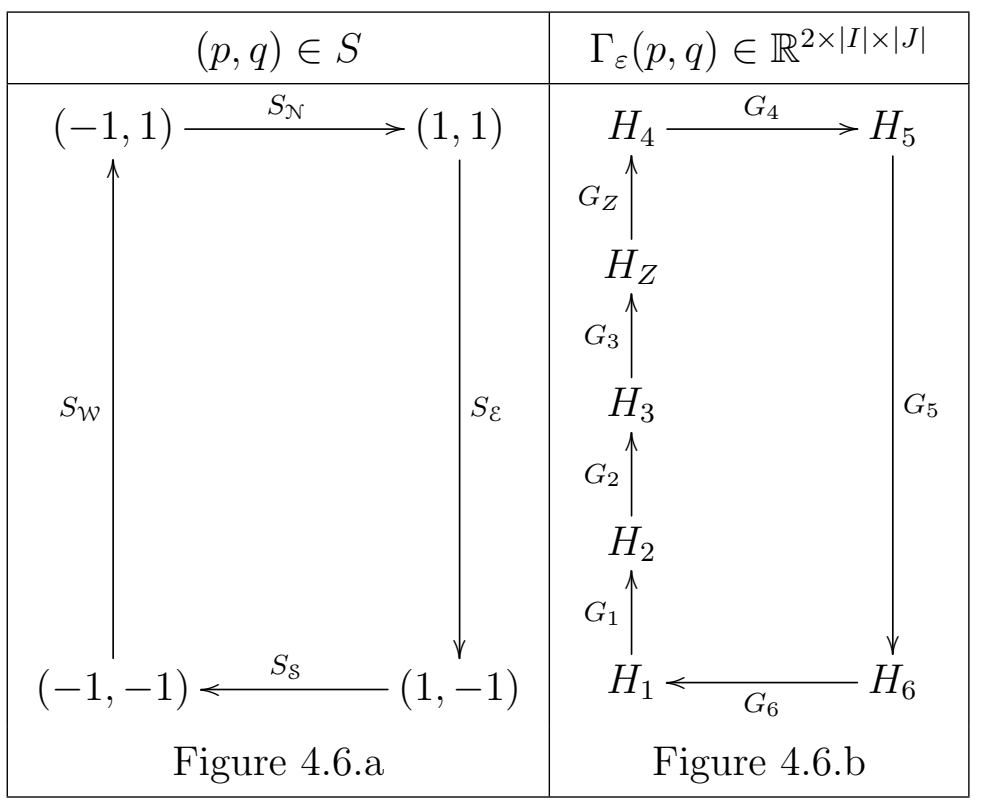

igure 4.6.a

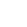

3


where $\vartheta$ is defined by

$\vartheta:=$\begin{tabular}{|c|c|c|c|}
\hline$A \backslash B$ & $L$ & $M$ & $R$ \\
\hline$L$ & 1,1 & 0,0 & $1,-1$ \\
\hline$M$ & 0,0 & $1,-1$ & $1,-1$ \\
\hline$R$ & $-1,1$ & $-1,-1$ & 0,0 \\
\hline
\end{tabular}

2 $\vartheta$ can be understood graphically:

\section{7}

$$
(R, M) \underset{\operatorname{Arc} 4}{(}(M, M)
$$$$
(-1,-1) \overleftarrow{\operatorname{Arc} 4}(1,-1)
$$

Figure 4.9.b

Figure 4.9.a

In addition, inequality (4.7) can be stated informally: For any equilibria of a game assigned to a point on $E$ via $\Gamma_{\varepsilon}$, the expected payoff under $\vartheta$ is not too far from the set of payoffs in equilibria on the edge opposite to $E$.

Proof: (Proof of Proposition 4.3.1) Property (i) is clear. (ii) follows (4.5), since each of the maps $G_{1}, \ldots, G_{6}, G_{Z}$ is $2 \varepsilon$-Lipshitz. (iii) holds since $\| G-$ $G_{0} \|_{\infty} \leq 2 \varepsilon$ whenever $G \in \Gamma_{\varepsilon}(S)=\left\{G_{j}(t) \mid j=1, \ldots, 6, Z, t \in[0,1]\right\}$.

(iv), as well, needs to be checked in each of the 7 segments of $S$ used in (4.5). Take, for example, $E=S_{\mathcal{N}}$; fix $(p, q) \in S_{\mathcal{N}}$. We have $q=1$ and hence $\Gamma_{\varepsilon}(p, q)$ is of the form $G_{4}(t)$ for some $t \in[0,1]$. Any equilibrium profile $(x, y)$ for $A, B$ in $G_{4}(t)$ lies on the arc $A_{4}$, and hence $E_{x \otimes y}(\vartheta)$ is a convex combination of $(1,-1)$ and $(-1,-1)$, i.e., is of the form $(s,-1)$, 
$-1 \leq s \leq 1$, and hence lies on $S_{\S}$. The other cases follow similarly, except for the arc $p=-1, \frac{1}{2} \leq q \leq 1$; here, one must use (4.4) together with the fact that for any two strategy pairs $x, y$ and $x^{\prime}, y^{\prime}$ for players $A, B$, we have

$$
\begin{aligned}
\mid E_{x \otimes y}[\vartheta] & -E_{x^{\prime} \otimes y^{\prime}}[\vartheta]|\leq| I|\cdot| J \mid \cdot\left\|x \otimes y-x^{\prime} \otimes y^{\prime}\right\|_{\infty} \cdot(\max \vartheta-\min \vartheta) \\
& =18\left\|x \otimes y-x^{\prime} \otimes y^{\prime}\right\|_{\infty}
\end{aligned}
$$

Q.E.D.

REMARK 4.3.2 For later purposes, we remark that the upper-semicontinuity of the Nash equilibrium correspondence implies that for each $\varepsilon>0$ there exists $\eta=\eta(\varepsilon)$ such that if $\left\|H-G_{0}\right\|_{\infty}<\eta$, then $N E(H)$ is contained in the $\varepsilon$-neighborhood of $N E\left(G_{0}\right)$.

\subsection{The Normal-Form Game}

In the appendix, we prove the following proposition, relying on a construction given in [24]:

Proposition 4.4.1 Let $I, J$ be finite sets, ${ }^{29}$ and let $Q: S \rightarrow \mathbb{R}^{2 \times I \times J}$ be a continuous and piecewise linear ${ }^{30}$ map to bimatrix games on these action sets. Then for some integer $M$, there exist 4 normal-form games on the set of players $A, B, \theta^{1}, \ldots, \theta^{M}$, denoted $\mathfrak{K}^{k}$ for $k \in\{1,-1\}^{2}$, such that:

1. A, $B$ have action spaces $I, J$ respectively; each $\theta^{j}$ has an action space $\{L, R\}$. The players $\left\{\theta^{1}, \ldots, \theta^{M}\right\}$ will be called auxiliary players.

2. The payoffs of $\theta^{1}, \ldots, \theta^{M}$ are not affected by the actions of $A, B$ in any of the games; let $\mathfrak{K}_{\Theta}^{k}$ denote the well-defined restriction of $\mathfrak{K}^{k}$ to the Players $\theta^{1}, \ldots, \theta^{M}$.

${ }^{29}$ The proposition also extends, with almost no change in the proof, to the case that $Q$ is a map to games with any finite set of players.

${ }^{30}$ I.e., piecewise linear on each edge of $S$.

ectaart.cls ver. 2006/04/11 file: stochastic_game_no_eq.tex date: March 27, 2013 
3. For $(p, q) \in[-1,1]^{2}$, let $\mathfrak{K}(p, q)$ (resp. $\mathfrak{K}_{\Theta}(p, q)$ ) denote the convex com-

1 bination of the $\left\{\mathfrak{K}^{k}\right\}_{k}$ (resp. $\left\{\mathfrak{K}_{\Theta}^{k}\right\}_{k}$ ), with weights given by $\left(\frac{1+p}{2}, \frac{1-p}{2}\right) \otimes$ $\left(\frac{1+q}{2}, \frac{1-q}{2}\right)$. If $(p, q) \in S$, and $a_{\theta}$ is an equilibrium in the game $\mathfrak{K}_{\Theta}(p, q)$, then the expected payoff matrix facing $A, B$, given by $\mathfrak{K}^{A, B}(p, q)\left(\cdot, a_{\theta}\right)$, is $Q(p, q)$.

4. For each $\varepsilon>0$, there is $\kappa=\kappa(\varepsilon)$ such that if $\left\|Q(p, q)-Q_{0}\right\|_{\infty} \leq \kappa$ for some $Q_{0}$, then

$$
\left\|\mathfrak{K}^{A, B}(p, q)\left(\cdot, a_{\theta}\right)-Q_{0}\right\|_{\infty} \leq \varepsilon, \forall(p, q) \in[-1,1]^{2}, \forall a_{\theta} \in N E\left(\mathfrak{K}_{\Theta}(p, q)\right)
$$

We now turn to our normal-form game. Fix $\varepsilon \leq \min \left[\frac{1}{4 \cdot|I| \cdot|J|}, \frac{1}{2} \kappa\left(\eta\left(\frac{1}{4 \cdot|I| \cdot|J|}\right)\right)\right]=$ $\min \left[\frac{1}{36}, \frac{1}{2} \kappa\left(\eta\left(\frac{1}{36}\right)\right)\right]$, where $\eta(\cdot)$ is defined in Remark 4.3.2 and $\kappa(\cdot)$ was defined in Proposition 4.4.1. The payoff depends on a parameter $\omega=\left(\omega^{C}, \omega^{D}\right) \in \mathbb{R}^{2}$ :

- The Players are $A, B, \theta^{1}, \ldots, \theta^{M}$, where $M$ corresponds to $Q:=\Gamma_{\varepsilon}$ as in Proposition 4.4.1 and $\Gamma_{\varepsilon}$ was constructed in Section 4.3, as well as an additional pair, Players $C, D$. (The auxiliary players $\theta^{1}, \ldots, \theta^{M}$ will not be discussed explicitly; the role they play is only through Proposition 4.4.1. Intuitively, one can think that the players $\theta^{1}, \ldots, \theta^{M}$ help provide 'communication' from $C, D$ to $A, B$, via their desire to react optimally to actions taken by the former pair.)

- As in Proposition 4.4.1, Players $A, B$ have action sets $I=J=$ $\{L, M, R\}$, and each player $\theta^{j}$ has action sets $\{L, R\}$; furthermore, Players $C, D$ each have action set $\{1,-1\}$.

- The payoff $r_{\omega}$ will be the sum of two payoffs, $r_{\omega}:=r_{1}+r_{2, \omega}$, defined separately as follows:

- The first payoff function $r_{1}$ does not depend on $\omega$, satisfies $r_{1}^{C, D}(a):=$ $\vartheta\left[a^{A, B}\right]$, where $\vartheta$ is defined in property (4.8) of Section 4.2, and the payoff to the other players is the same as in the game of Proposition 4.4.1 when the profile $a^{-\{C, D\}}$ is played and the choice $a^{C, D} \in$ 
$\{+1,-1\}^{2}$ is made by Nature; namely,

$$
r_{1}^{C, D}(a):=\vartheta\left[a^{A, B}\right], \quad r_{1}^{-\{C, D\}}(a)=\mathfrak{K}^{a^{C, D}}\left(a^{-\{C, D\}}\right)
$$

- The second payoff function $r_{2, \omega}$ depends on $\omega$. It gives a payoff of 0 to all players other than $C, D$ : That is, $r_{2, \omega}^{-\{C, D\}} \equiv 0$. To players $C, D$, $r_{2, \omega}$ is dependent only on $a^{C, D}$ and is given by:

$r_{2, \omega}^{C, D}(a)=$\begin{tabular}{|c|c|c|}
\hline$C \backslash D$ & 1 & -1 \\
\hline 1 & $\omega^{C}, \omega^{D}$ & $\frac{1}{2} \omega^{C}, \frac{1}{2} \omega^{D}$ \\
\hline-1 & $\frac{1}{2} \omega^{C}, \frac{1}{2} \omega^{D}$ & 0 \\
\hline
\end{tabular}

(In the stochastic game - which is built around this game normal-form game - that we will define, players $C, D$ control the transitions but do not influence their own stage payoffs, and $\omega^{C, D}$ will be the expected continuation payoff for these players if the game does not enter its quitting state.)

For each $(p, q) \in S$, let $a_{p, q}$ be an equilibrium profile in the game with payoff $r_{\omega}$ for the players $A, B, \theta^{1}, \ldots, \theta^{M}$ when Players $C, D$ are restricted to playing $b_{p, q}:=\left(\frac{1+p}{2}, \frac{1-p}{2}\right) \otimes\left(\frac{1+q}{2}, \frac{1-q}{2}\right)$; that is $a_{p, q}$ is an equilibrium in $r_{1}^{-\{C, D\}}\left(\cdot, b_{p, q}\right)=r_{\omega}^{-\{C, D\}}\left(\cdot, b_{p, q}\right)$. We will continue formally below, but we give a geometric image of where we are heading: Property (3) of Proposition 4.4.1, applied to the mapping $Q:=\Gamma_{\varepsilon}$ which has the properties given in Proposition 4.3.1, together with Figure 4.9 gives the following relationship between $p, q$ and the payoff in $r_{1}$ to $C, D$ under the profile $a_{p, q}, r_{1}^{C, D}\left(a_{p, q}, b_{p, q}\right)$ :

(1)

(1)




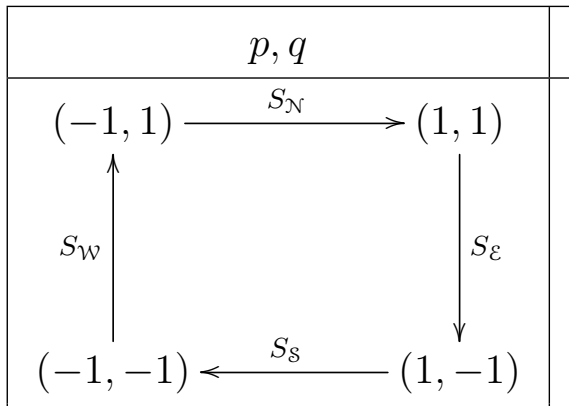

Figure 4.11.a
The diagram is to be understood in the following way: as the point $(p, q)$ goes around the square, the payoff $r_{1}^{C, D}\left(a_{p, q}, b_{p, q}\right)$ (which is not uniquely determined) must also go 'around' the square 'close to it' - at a distance of at most $18 \varepsilon$ from the edge opposite the edge on which $(p, q)$ lies, because of (4.7). Formally:

Proposition 4.4.2 Let $\omega \in \mathbb{R}^{2}$, and let a be an equilibrium profile in the game $r_{\omega}$. Denote $p=2 a^{C}[1]-1, q=2 a^{D}[1]-1$. Then:

1. If $\omega^{C}>0$, then $p=1$; if $\omega^{C}<0$, then $p=-1$. The same holds for $q$ w.r.t. $\omega^{D}$.

2. If $\omega^{C}>0$, then $r_{1}^{C}(a) \leq-\frac{1}{2}$. If $\omega^{C}<0$, then $r_{1}^{C}(a) \geq \frac{1}{2}$. Similarly, if $\omega^{D}>0$, then $r_{1}^{D}(a) \leq-\frac{1}{2}$, and if $\omega^{D}<0$, then $r_{1}^{D}(a) \geq \frac{1}{2}$.

3. Let $H$ be the expected matrix facing players $A, B$; that is, $H=r_{\omega}^{A, B}\left(\cdot, a^{-\{A, B\}}\right)$. Then $\left\|H-G_{0}\right\|_{\infty}<\eta\left(\frac{1}{4}\right)$ (regardless of the values of $\omega^{C}, \omega^{D}$; this includes the case where one or both are 0$)$, and $r_{1}^{C, D}(a) \neq 0$.
Proof: The first part follows simply from the definition of $r_{2, \omega}$ and since $r_{\omega}^{C, D}-r_{2, \omega}^{C, D}$ is independent of the actions of players $C, D$. For the second part, take, for example, the case $\omega^{C}>0$, which, by the first part, implies $p=1$. Since $a \in N E(Q(p, q))=N E\left(\Gamma_{\varepsilon}(p, q)\right) \in \cup_{x \in E} N E\left(\Gamma_{\varepsilon}(x)\right)$, where $E=\{1\} \times[-1,1]=S_{\mathcal{E}}$ in this case, it follows from (4.7) that, $\left\|r_{1}^{C, D}(a)-\left(-S_{\mathcal{E}}\right)\right\|_{\infty}=\left\|E_{a^{A, B}}[\vartheta]-S_{\mathcal{W}}\right\|_{\infty}<18 \varepsilon \leq \frac{1}{2}$ 
and hence $r_{1}^{C}(a) \leq-1+\frac{1}{2}=-\frac{1}{2}$. The case $\omega^{C}<0$, as well as the cases $\omega^{D}>0, \omega^{D}<0$, follow similarly.

For the last part, first note that by property (iii) of Proposition 4.3.1, $\left\|Q(p, q)-G_{0}\right\|_{\infty}<2 \varepsilon \leq \kappa\left(\eta\left(\frac{1}{4|I| \cdot J \mid}\right)\right)$ for all $(p, q) \in S$. By property (4) of Proposition 4.4.1, we see that $\left\|H-G_{0}\right\|_{\infty}<\eta\left(\frac{1}{4|I| \cdot J \mid}\right)$, which, by definition ${ }^{31}$ of $\eta$, implies that there is an equilibrium $b$ of $G_{0}$ with $\|a-b\|_{\infty}<\frac{1}{4|I| \cdot|J|}$. Since for any equilibrium $b$ of $G_{0}$ we have $E_{b}[\vartheta] \in S$, we see by (4.10) that $r_{1}^{C, D}(a)=\vartheta\left[a^{A, B}\right]$ is in the $\frac{1}{2}$-neighborhood of the square $S$, and in particular, $r_{1}^{C, D}(a) \neq 0$.

Q.E.D.

\subsection{The Stochastic Game}

The stochastic game has the following components:

- The players are $\mathcal{P}=\left\{A, B, C, D, \theta^{1}, \ldots, \theta^{M}\right\}$ as in Section 4.4, along with the actions sets given there.

- The state space $\Omega$ is $[0,1]$, with the Borel $\sigma$-algebra.

- The payoff function $r(s, \cdot)$ in state $s$ is given by $(1-s) r_{1}(\cdot)$, where $r_{1}$ is defined in Section 4.4. Note that $r(1, \cdot) \equiv 0$.

- The transitions $q(t, a)$ are controlled by Players $C, D$ and are given by:

$$
q(t, a)=(1-\zeta(1-t)) \delta_{1}+\zeta(1-t) \cdot \tilde{q}(t, a)
$$

where $0<\zeta \leq 1$ is fixed and satisfies

$$
\frac{\zeta \cdot\|r\|_{\infty}}{1-\zeta}<\frac{1}{2}
$$

and

$\tilde{q}(t, a)=$\begin{tabular}{|c|c|c|}
\hline$C \backslash D$ & $L$ & $R$ \\
\hline$L$ & $U(t, 1)$ & $\frac{1}{2} U(t, 1)+\frac{1}{2} \delta_{1}$ \\
\hline$R$ & $\frac{1}{2} U(t, 1)+\frac{1}{2} \delta_{1}$ & $\delta_{1}$ \\
\hline
\end{tabular}

${ }^{31}$ See Remark 4.3.2.

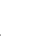

(1)

(1)

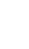

(1)

(1)

8

9

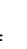

5

16

7

8


where $U(a, b)$ is the uniform distribution on $[a, b]$, and $\delta_{c}$ is the Dirac measure at $c$; we interpret $U(1,1)=\delta_{1}$. Note that 1 is an absorbing state.

- $\beta \in(0,1)$ is a discount factor.

REMARK 4.5.1 It is clear that all transitions are absolutely continuous w.r.t. $\frac{1}{2} U(0,1)+\frac{1}{2} \delta_{1}$, and hence the game satisfies ACC. Furthermore, if one desires absolute continuity w.r.t. a non-atomic measure, we can make the following alteration: Since 1 is an absorbing state with payoff 0 to all, one could replace $\{1\}$ with a continuum $[1,2]$ of absorbing states with payoff 0 to all, replacing $\delta_{1}$ by $U(1,2)$ throughout, and hence all transitions would be absolutely continuous w.r.t the Lebesgue measure.

By way of contradiction, fix a stationary equilibrium $\sigma$. Recall the notations $\gamma_{\sigma}$ and $X_{\sigma}$ from Section 2. We will denote for $j=C, D, V^{j}=\gamma_{\sigma}^{j}$ and $W^{j}(t)=\int_{t}^{1} V^{j}(s) d s$. For $j=C, D,(2.5)$ becomes

$$
V^{j}(t)=X_{\sigma}^{j}(t, \sigma(t))=r^{j}(t, \sigma(t))+\beta \zeta(1-q(\{1\} \mid t, \sigma(t))) W^{j}(t) .
$$

From the definition of the payoffs, it follows that:

LEMMA 4.5.2 For $0 \leq t \leq 1$,

$$
X_{\sigma}(t, \cdot)=(1-t) r_{\omega(t)}(\cdot)+\xi_{\sigma}(t, \cdot)
$$

where $r_{\omega}$ is defined in Section $4.4, \xi_{\sigma}^{C} \equiv \xi_{\sigma}^{D} \equiv 0$,

$$
\xi_{\sigma}^{-\{C, D\}}(t, a)=\beta \zeta \cdot(1-q(\{1\} \mid t, a)) \cdot \int_{t}^{1} \gamma_{\sigma}^{-\{C, D\}}(t) d t
$$

and $\omega$ is given by

$$
\omega(t)=\left(\omega^{C}(t), \omega^{D}(t)\right):=\zeta \beta \cdot \int_{t}^{1} \gamma_{\sigma}^{\{C, D\}}(s) d q(t, \sigma(t))=\zeta \beta \cdot\left(W^{C}(t), W^{D}(t)\right) .
$$

Lemma 4.5.3 For $\omega$ as in Lemma 4.5.2, we have $\|\omega\|_{\infty}<\frac{1}{2}$. 
34

Proof: Since $q([0,1) \mid \cdot) \leq \zeta$, it follows for $j \in\{C, D\}$,

$$
\left|V^{j}\right| \leq \sum_{j=1}^{\infty}\|r\|_{\infty} \cdot \zeta^{j-1}=\frac{\|r\|_{\infty}}{1-\zeta}
$$

and hence (4.12) implies that

$$
\left|\omega^{j}\right|=\left|\zeta \beta \cdot W^{j}\right|<\beta \frac{\zeta \cdot\|r\|_{\infty}}{1-\zeta}<\frac{1}{2}
$$

Q.E.D.

It is immediate that:

LEMMA 4.5.4 Let $g_{1}, g_{2}$ be two payoff functions on the same player set, such that for any Player $p$ and any pair of pure action profiles $a, b$ that differ (at most) in Player p's action,

$$
g_{1}^{p}(a)-g_{1}^{p}(b)=g_{2}^{p}(a)-g_{2}^{p}(b)
$$

Then the set of Nash equilibria under $g_{1}$ is the same as the set of Nash equilibria under $g_{2}$.

Note that under $\xi_{\sigma}(t, \cdot)$, each player's payoff is independent of his own action. Combining this observation with Lemma 4.5.4 (where $g_{1}(\cdot)=X_{\sigma}(t, \cdot)$ and $\left.g_{2}(\cdot)=(1-t) r_{\omega(t)}(\cdot)\right)$, Lemma 4.5.2, and Proposition 4.4.2, we deduce that for each $t \in[0,1]$ :

- If $W^{C}(t)>0$ (resp. $\left.<0\right), r^{C}(t, \sigma(t)) \leq-\frac{1}{2}$ (resp. $\left.\geq \frac{1}{2}\right)$.

- If $W^{D}(t)>0($ resp. $<0), r^{D}(t, \sigma(t)) \leq-\frac{1}{2}\left(\right.$ resp. $\left.\geq \frac{1}{2}\right)$.

- Regardless of the values of $W^{C}(t), W^{D}(t)$,

$$
r^{C}(t, \sigma(t)) \neq 0 \text { or } r^{D}(t, \sigma(t)) \neq 0
$$

Using these observations, we can further deduce that for each $t \in[0,1]$ : 
- Since Lemma 4.5.3 implies that:

$$
\left\|r^{C, D}(t, \cdot)-X_{\sigma}^{C, D}(t, \cdot)\right\|_{\infty}=\|\omega(t)\|_{\infty}<\frac{1}{2}
$$

it follows from (4.13) that if $W^{C}(t)>0($ resp. $<0)$, then $V^{C}(t)<0$ (resp. $>0$ ), and similarly for $V^{D}$ w.r.t. $W^{D}$.

- We deduce that for at least one $j \in\{C, D\}, V^{j}(t) \neq 0$ : If $W^{C}(t)=$ $W^{D}(t)=0$, we deduce this from (4.14) and (4.13), while otherwise it follows from the case above.

Furthermore, it is known that for a.e. $t, \frac{d W^{j}}{d t}(t)=-V_{j}(t)$ for $j=C, D$. Define $G=\left(W^{C}\right)^{2}+\left(W^{D}\right)^{2}$. Our conclusions show that for at least one $j \in\{C, D\}, W^{j}$ is non-zero somewhere (otherwise, we would have $V^{1} \equiv$ $V^{2} \equiv 0$ ), and hence $G$ is not uniformly 0 . Furthermore, it holds a.e. that

$$
G^{\prime}=2 \cdot W^{c} \cdot \frac{d W^{C}}{d t}+2 \cdot W^{D} \cdot \frac{W^{D}}{d t} \geq 0
$$

$G$ is absolutely continuous, because both $W^{C}, W^{D}$ are absolutely continuous (and hence also bounded.) Therefore, since $G^{\prime} \geq 0$ a.e. and $G$ is positive at some point, we deduce that $G(1)>0$, a contradiction since $G(1)=0$.

\subsection{Necessary Components of Construction}

As has been discussed in Section 1, the question of existence of stationary equilibrium in discounted stochastic games under the ACC assumption has attracted much attention. Much of this attention has resulted from the particular models used in particular economic interactions, such as capital accumulation, models with heterogeneous shocks, and others. Future research will undoubtedly include attempts to formulate very general conditions under which such equilibria do or do not exist in these models. Hence, we briefly mention here (without proof) what components - or, more specifically, what anomalies in the manifold of Nash equilibria - are really required 
for the construction of a basic normal-form game which satisfies the properties of Proposition 4.4.2.

The multi-player normal-form game could be built around a 'base' normalform game $G_{0}$ (with any finite number of players) with the following properties:

(1) The set of equilibria $N E_{0}=N E\left(G_{0}\right)$ contains $^{32}$ a unique hyperstable set $H_{0}$. By a hyperstable set, defined in [21], we mean a set that is minimal w.r.t. the following property: for any $\varepsilon>0$, there is $\delta>0$ such that the equilibria of any game $G^{\prime}$ that is in a $\delta$-neighborhood of a game $G$ that is equivalent ${ }^{33}$ to $G_{0}$ are in an $\varepsilon$-neighborhood of $H_{0}$.

(2) $H_{0}$ is connected but not nulhomotopic.

(3) Furthermore ${ }^{34}$ there exists:

- For some $n \in \mathbb{N}$, a continuous semi-algebraic ${ }^{35}$ injection $\psi: C^{n} \rightarrow$ $H_{0}$, which is not nulhomotopic in $H_{0}$, where $C^{n}$ is the boundary of the $n+1$-cube: $C^{n}=\left\{x \in \mathbb{R}^{n+1} \mid \exists i \in\{1, \ldots, n+1\}\right.$ s.t. $x_{i} \in$ $\{1,-1\}\}$.

- A semi-algebraic retract $\rho: N E_{0} \rightarrow \psi\left(C^{n}\right)$.

- For all $\varepsilon>0$, a semi-algebraic mapping $\Gamma_{\varepsilon}$ from $C^{n}$ to the $\varepsilon$ neighborhood of $G_{0}$, such that for each edge $E$ of $C^{n}$ (i.e., $E$ is of the form $\left\{x \in C^{n} \mid x_{i}=q\right\}$ for some $i$ and some $\left.q \in\{-1,1\}\right)$, any equilibrium of any game in $\Gamma_{\varepsilon}(E)$ is in an $\varepsilon$-neighborhood of $\rho^{-1}(\psi(-E))$.

${ }^{32} N E_{0}$ may contain other components which are not hyperstable.

${ }^{33}$ Two games are equivalent if they have the same reduced form, where the reduced form is achieved by eliminating actions that are payoff-equivalent to a convex combination of other actions.

${ }^{34}$ It is not clear if some of the components of Property (3) already follow from Property (2); in view of Remark 4.6.1, this is equivalent to saying that it is not clear what regularity conditions the manifold of Nash equilibria possess.

${ }^{35}$ See, e.g., [4]. 
REMARK 4.6.1 Property (3) can be viewed as a regularity condition on

1 the manifold of Nash equilibria near the game $G_{0}$.

In our case, in which $n=1$, can take $\psi: S\left(=C^{2}\right) \rightarrow H_{0}$ as in Figure 4.9.a, and $\rho$ to be the identity; $\Gamma_{\varepsilon}$ was defined in (4.5).

5. APPENDIX: PIECEWISE LINEAR GAMES ON THE SQUARE

In this section we prove Proposition 4.4.1. We recall the following proposition from [24] (we use the notations and conventions - in particular, that all metrics are w.r.t. the supremum norm - introduced in Section 4.2):

Proposition 5.0.2 Let $f:[a, b] \rightarrow(0,1)$ be a continuous, piecewise linear function. Then there exist ${ }^{36}$ an integer $N>0$ and two normal-form games, $\mathfrak{G}^{L}$ and $\mathfrak{G}^{R}$, on the set of players ${ }^{37} A, B, \alpha^{1}, \ldots, \alpha^{N-1}$, each with action space $\{L, R\}$, such that for any $p \in[a, b]$, denoting

$$
\mathfrak{G}(p):=\frac{p-a}{b-a} \cdot \mathfrak{G}^{L}+\frac{b-p}{b-a} \mathfrak{G}^{R}
$$

it holds that in any equilibrium of $\mathfrak{G}(p)$, Players $A, B$ play the mixed action profile $(f(p), 1-f(p)) \times(f(p), 1-f(p))$.

REMARK 5.0.3 The construction above has other properties:

(i) The payoffs of each of the $\left(\alpha^{j}\right)$ - these players will be referred to as auxiliary players - are independent of the actions of any other player; hence, we can refer to the matrix $G(p)$, which is the expected matrix facing players $A, B$ when each of the $\alpha^{j}$ plays an optimal action; this turns out to be well-defined, as when any $\alpha^{j}$ are indifferent in $\mathfrak{G}(p)$ for some $p$, any choices yield the same expected payoffs for players $A, B$.

${ }^{36} N$ is the number of segments into which $[a, b]$ has to be divided into in order for $f$ to be linear in each segment.

${ }^{37}$ When $N=0$, the set of players is just $A, B$. 
(ii) In fact, by construction, $G(p)$ is uniquely determined by the value of $f$ at $p$, as it turns out that we have explicitly,

$$
G(p)=\bar{G}(f(p)), \text { where } \bar{G}(t)=\begin{array}{|c|c|c|}
\hline & L & R \\
\hline L & 1,-1 & 1-4 t, 3-4 t \\
\hline R & 4 t-3,4 t-1 & 1,-1 \\
\hline
\end{array}
$$

(iii) The construction there also shows that if $L$ is a Lipschitz constant of $f$, and $\left\|f-f_{0}\right\|_{\infty} \leq \kappa$ for some $f_{0}, \kappa \in \mathbb{R}$, then $\left\|\left(\mathfrak{G}^{k}\right)^{A, B}-\bar{G}\left(f_{0}\right)\right\|_{\infty} \leq$ $(b-a) L \kappa$ for $k \in\{L, R\}$.

Proposition 5.0.4 Let $S$ be the boundary of the square:

$$
S=\{(p, q) \mid-1 \leq p, q \leq 1,(|p|=1) \vee(|q|=1)\}
$$

and let $g: S \rightarrow(0,1)$ be a continuous and piecewise linear ${ }^{38}$ map. Then for some integer $K$, there exists four normal form games on the set of players $A, B, \gamma, \delta, \beta^{1}, \ldots, \beta^{K}$, denoted $\mathfrak{H}^{k}$ for $k \in\{1,-1\}^{2}$, such that:

- $A, B$ and also each of the $\left(\beta^{j}\right)$ has the action set $\{L, R\}$, and for each $j$ and each $k \in\{1,-1\}^{2}$, the payoff of $\beta^{j}$ in $\mathfrak{H}^{k}$ is independent of any other player's action. ${ }^{39}$

- $\gamma, \delta$ have action set $\{1,-1\}$.

- If Nature chooses $k \in\{1,-1\}^{2}$ with distribution ${ }^{40}\left(\frac{1+p}{2}, \frac{1-p}{2}\right) \otimes\left(\frac{1+q}{2}, \frac{1-q}{2}\right)$, $(p, q) \in S$, and $\beta^{1}, \ldots, \beta^{k}$ all play best responses $a^{\beta^{1}}, \ldots, a^{\beta^{k}}$ in the game

$$
\mathfrak{H}(p, q)=\sum_{k \in\{1,-1\}^{2}}\left(\left(\frac{1+p}{2}, \frac{1-p}{2}\right) \otimes\left(\frac{1+q}{2}, \frac{1-q}{2}\right)\right)[k] \cdot \mathfrak{H}^{k}
$$

\footnotetext{
${ }^{38}$ That is, piecewise linear on each of the four edges of $S$.
}

${ }^{39}$ This is unlike the $\left(\theta^{j}\right)$ of Proposition 4.4.1, which we later prove using Proposition 5.0.4; the payoffs of $\theta^{1}, \ldots, \theta^{M}$ can be affected by each other's actions.

${ }^{40}$ Recall that $(\phi, 1-\phi)$ denotes the probability distribution choosing 1 with probability $\phi$, and choosing -1 with probability $1-\phi$.

ectaart.cls ver. 2006/04/11 file: stochastic_game_no_eq.tex date: March 27, 2013 
then the expected game facing $A, B$, denoted

1

$$
H(p, q):=\left(\mathfrak{H}(p, q)\left(\cdot, a^{\beta^{1}}, \ldots, a^{\beta^{k}}\right)\right)^{A, B}
$$

is well-defined, ${ }^{41}$ and its unique equilibrium is $(g(p), 1-g(p)) \times(g(p), 1-$ $g(p))$.

- If $L$ is a Lipshitz constant of $g$ (on each edge) and $\left|g(p, q)-g_{0}\right|<\varepsilon$ for all $p, q \in S$, then there is $H_{0}$ such that

$$
\left\|\left(\mathfrak{H}^{k}\right)^{A, B}(a)-H_{0}\right\|_{\infty} \leq 2 L \varepsilon, \forall a, \forall k \in\{-1,1\}^{2}
$$

Proof: We denote the vertices of the square $S$ by

$$
V_{-,+}=(-1,1) \stackrel{S_{\mathcal{N}}}{\longrightarrow} V_{+,+}=(1,1)
$$

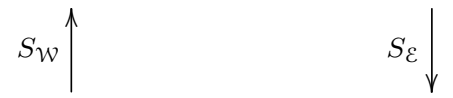$$
V_{-,-}(-1,-1) \longleftarrow_{S_{W}} V_{+,-}=(1,-1)
$$

For $i \in\{-,+\}^{2}$, let $i+$ be such that $V_{i+}$ follows $V_{i}$ in the clockwise orientation. For $i \in\{-,+\}^{2}$, let $g_{i}:[-1,1] \rightarrow \mathbb{R}$ be the function of one parameter which is the restriction of $g$ to the arc extending clockwise from $V_{i}$; that is, $g_{i}(0)=g\left(V_{i}\right)$ and $g_{i}(1)=g\left(V_{i^{+}}\right)$, and $g_{i}$ 'behaves' like $g$ on the arc from $V_{i}$ to $V_{i+}$. For example, $g_{+,+}(t)=g(-t, 1)$, so $g_{+,+}(-1)=g\left(V_{+,+}\right)$, $g_{+,+}(1)=g\left(V_{+,-}\right)$.

For $j \in\{-,+\}^{2}$, let $N_{j}$ correspond to $g_{j}$ as in Proposition 5.0.2. Then let $K=\sum_{j}\left(N_{j}-1\right)$; and also treat $K$ as the set $\{1, \ldots, K\}$ partitioned into subsets $N_{V_{ \pm, \pm}}$of sizes $N_{ \pm, \pm}-1$. For each $k \in\{-,+\}^{2}$, let $\mathfrak{G}_{k}^{m}, m=L, R$, be the two games that correspond to $g_{k}$ on the set of players $A, B, \beta^{1}, \ldots, \beta^{K}$, as in Proposition 5.0.2 (the auxiliary players which were there denoted $\left(\alpha^{j}\right)_{j<N_{V_{k}}}$

${ }^{41}$ I.e., if some $\beta^{k}$ are indifferent between actions, it doesn't matter for players $A, B$ which they choose. 
are now $\left(\beta^{j}\right)_{j \in N_{V_{k}}}$ - i.e., $\left(\beta^{j}\right)_{j \in K}=\cup_{k \in\{-,+\}^{2}}\left(\alpha^{j}\right)_{j<N_{V_{k}}}$, where the union is disjoint - and $\beta^{j}$ is given a payoff of 0 in $\mathfrak{G}_{k}^{m}$ for each $j \notin N_{V_{k}}$.) For each $k \in$ $\{-,+\}^{2}$, let $G_{k}(t)$ denote the corresponding expected matrix to $A, B$ when auxiliary players play optimally in $\mathfrak{G}_{k}(t)$; as we have mentioned in property (i) of Remark 5.0.3, this bimatrix game is well defined, $G_{k}(t)=\bar{G}\left(g_{k}(t)\right)$. Hence, we have $G_{k}(1)=\bar{G}\left(g_{k}(1)\right)=\bar{G}\left(g\left(V_{k+}\right)\right)=\bar{G}\left(g_{k+}(-1)\right)=G_{k+}(-1)$.

We can now define $\left(\mathfrak{H}^{k}\right)_{k}$ from the $\left(\mathfrak{G}_{k}\right)_{k}$ as follows. First, define the payoffs to $\gamma, \delta$. For each of these players, the payoff is determined only by $k$ and his own action. The payoffs to $\gamma$ in the various games are given by the following table:

\begin{tabular}{|c|c|c|c|c|}
\hline & $k=(1,1)$ & $k=(1,-1)$ & $k=(-1,-1)$ & $k=(-1,1)$ \\
\hline$\gamma$ plays 1 & 0 & 1 & 0 & -1 \\
\hline$\gamma$ plays -1 & 0 & -1 & 0 & 1 \\
\hline
\end{tabular}

and the payoffs to $\delta$ by

\begin{tabular}{|c|c|c|c|c|}
\hline & $k=(1,1)$ & $k=(1,-1)$ & $k=(-1,-1)$ & $k=(-1,1)$ \\
\hline$\delta$ plays +1 & 1 & 0 & -1 & 0 \\
\hline$\delta$ plays -1 & -1 & 0 & 1 & 0 \\
\hline
\end{tabular}

The diagram below describes the best-replies of $\gamma, \delta$ when Nature chooses $k \in\{+1,-1\}^{2}$ via the distribution $(p, 1-p) \otimes(q, 1-q)$ (with $\gamma, \delta$, and Nature making their choices simultaneously). In the diagram, this (mixed) choice of Nature is represented by the point with coordinates $(2 p-1,2 q-1)$, and the best-reply profile of $\gamma, \delta$ depends on which of the four regions in the square Nature chooses. 


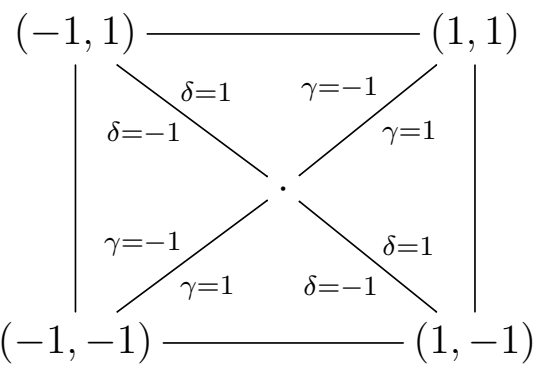

On one diagonal, $\gamma$ will be indifferent; on the other, $\delta$ will be. More formally, we deduce from the payoffs of $\gamma, \delta$ defined above that:

- If $p>q($ resp. $<)$, then $\gamma$ strongly prefers to play +1 (resp. -1 ).

- If $p>1-q($ resp. $<)$, then $\delta$ strongly prefers to play +1 (resp. -1 ). Now, we define the payoffs to Players $A, B, \beta^{1}, \ldots, \beta^{K}$. Given the choice of Nature $k \in\{+1,-1\}^{2}$, the actions of $\gamma$ and $\delta$ determine which game $A, B, \beta^{1}, \ldots, \beta^{K}$ face, as depicted in the following table (* denotes an arbitrary action):

\begin{tabular}{|c|c|c|c|}
\hline Game & Action of $\gamma$ & Action of $\delta$ & Game Facing $A, B, \beta^{1}, \ldots, \beta^{K}$ \\
\hline $\mathfrak{H}^{1,1}$ & -1 & $*$ & $\left(\mathfrak{G}_{-,+}\right)^{R}$ \\
\hline $\mathfrak{H}^{1,1}$ & 1 & $*$ & $\left(\mathfrak{G}_{+,+}\right)^{L}$ \\
\hline $\mathfrak{H}^{1,-1}$ & $*$ & -1 & $\left(\mathfrak{G}_{+,+}\right)^{R}$ \\
\hline $\mathfrak{H}^{1,-1}$ & $*$ & 1 & $\left(\mathfrak{G}_{+,-}\right)^{L}$ \\
\hline $\mathfrak{H}^{-1,-1}$ & -1 & $*$ & $\left(\mathfrak{G}_{+,-}\right)^{R}$ \\
\hline $\mathfrak{H}^{-1,-1}$ & 1 & $*$ & $\left(\mathfrak{G}_{-,-}\right)^{L}$ \\
\hline $\mathfrak{H}^{-1,1}$ & $*$ & -1 & $\left(\mathfrak{G}_{-,-}\right)^{R}$ \\
\hline $\mathfrak{H}^{-1,1}$ & $*$ & 1 & $\left(\mathfrak{G}_{-,+}\right)^{L}$ \\
\hline
\end{tabular}

Since we have already noticed that $G_{k}(1)=G_{k^{+}}(0)$ for all $k$, one can verify that these games do indeed satisfy that for any $(p, q) \in S$, the unique equilibrium of the expected game facing $A, B$ is $(g(p), 1-g(p)) \times(g(p), 1-$ 
$g(p))$. For example, if $(p, q) \in S_{\mathcal{N}}$ is an internal point of the edge, then $q=1$ and $-1<p<1$. Then we will have in equilibrium $\gamma=-1, \delta=1$, and hence,

$$
\mathfrak{H}^{-\{\gamma, \delta\}}(p, q)(\cdot, \gamma=-1, \delta=1)=\mathfrak{H}^{-\{\gamma, \delta\}}(p, 1)(\cdot, \gamma=-1, \delta=1)
$$$$
=\frac{1+p}{2}\left(\mathfrak{H}^{1,1}\right)^{-\{\gamma, \delta\}}(\cdot, \gamma=-1, \delta=1)
$$$$
+\frac{1-p}{2}\left(\mathfrak{H}^{-1,1}\right)^{-\{\gamma, \delta\}}(\cdot, \gamma=-1, \delta=1)
$$$$
=\frac{1-p}{2} \mathfrak{G}_{-,+}^{L}+\frac{1+p}{2} \mathfrak{G}_{-,+}^{R}
$$

and hence, by definition of $\mathfrak{G}_{-,+}^{L}, \mathfrak{G}_{-,+}^{R}$, and of $H(p, q)$, we have

$$
H(p, q)=g_{+,+}(p)=g(p, 1)=g(p, q)
$$

A similar arguments works for the internal points on any edge; the vertices of $S$ are simpler to verify.

Finally, the last property, given in (5.1), follows from part (iii) of Remark 5.0 .3 .

Q.E.D.

Proof: (of Proposition 4.4.1) It suffices to prove the case ${ }^{42} 0<Q<1$; otherwise, we will adjust $Q$ to satisfy this normalization via an affine transformation, and then apply to the inverse affine transformation to the game we derive. For each $(p, i, j) \in\{A, B\} \times I \times J$, let $Q_{p, i, j}: S \rightarrow(0,1)$ be the corresponding component of $Q$; and for each such piecewise linear function, let $\left(\mathfrak{H}_{p, i, j}^{k}\right)_{k \in\{1,-1\}^{2}}$ be the four corresponding games from Proposition 5.0.4, on the set of players $P_{p, i, j}:=\left\{A_{p, i, j}, B_{p, i, j}, \gamma_{p, i, j}, \delta_{p, i, j}, \beta_{p, i, j}^{1} \ldots, \beta_{p, i, j}^{N_{p, i, j}}\right\}$ for some $N_{p, i, j}$. When Nature chooses $k \in\{-1,1\}^{2}$, each set of players $P_{p, i, j}$ plays $\mathfrak{H}_{p, i, j}^{k}$, and the payoff to Player $A$ (resp. $B$ ) when action profile $(i, j)$ is played is 1 if $A_{p, i, j}$ plays $L$, and 0 if he plays $R$. We then take $\theta^{1}, \ldots, \theta^{M}$

\footnotetext{
${ }^{42}$ The strong inequalities refer to all coordinates.
} 
to be some enumeration of $\cup_{p, i, j} P_{p, i, j}$. Property (4) follows from the upper-

1

semicontinuity of the equilibrium correspondence, and by (5.1). Q.E.D.

\section{REFERENCES}

[1] Amir, R. (1991) On stochastic games with uncountable state and action spaces, in Stochastic Games and Related Topics, (T. E. S. Ragahavan, T. S. Ferguson, T. Parthasarathy, and O. J. Vrieze, Eds.), Boston, Kluwer Academic, pp. 149-159.

[2] Amir, R. (1996), Continuous Stochastic Games of Capital Accumulation with Convex Transitions, Games Econom. Behav., 15, 111-131.

[3] Amir, R. (2003), Stochastic Games in Economics and Related Fields: An Overview, in Stochastic Games and Applications, NATO Adv. Sci. I. C-MAT (A. Neyman and S. Sorin, Eds.), Vol. 570, Dordrecht, Kluwer Academic Publishers, Chapter 30, pp. 455-470.

[4] Bochnak, J., Coste, M., And Roy, M.F. (1998), Real Algebraic Geometry, Springer-Verlag, Berlin.

[5] Basar, T. And Olsder, G. (1999), Dynamic Noncooperative Game Theory, Philadelphia, SIAM Classics.

[6] Beggs, A. And Klemperer, P. (1992), Multi-Period Competition with Switching Costs, Econometrica, 60, 651-666.

[7] Bertsekas, D. And Shreve, S. (1996) Stochastic Optimal Control: The Discrete Time Case, Belmont, MA, Athena Scientific.

[8] Chakrabarti, S.K. (1999), Markov Equilibria in Discounted Stochastic Games. J. Econ. Theory, 85, 294-327.

[9] Duffie, D., Geanakoplos, J., Mas-Colell, A., and McLenNAN, A. (1994), Stationary Markov Equilibria, Econometrica, 62, 745781.

[10] Duggan, J. (2012) Noisy Stochastic Games, Econometrica, 80, 20172045 . 
[11] Dutta, P. and Sundaram, R. (1998), The Equilibrium Existence Problem in General Markovian Games, in Organizations with Incomplete Information (M. Majumdar, Ed.), Cambridge, Cambridge University Press.

[12] Federgruen, A., On $N$-Person Stochastic Games with Denumerable State Space, Adv. Appl. Probab., 10, 452-471.

[13] Fink, A.M. (1964), Equilibrium in Stochastic $n$-Person Games, Hiroshima J. Sci., 28, 89-93.

[14] Forges, F. (1986), An Approach to Communication Equilibria, Econometrica, 54, 1375-1385.

[15] Guesnerier, R., And Woodford, M. (1992), Endogenous Fluctuations, in Advances in Economic Theory, Vol. II, (J.J. Laffont, Ed.), Cambridge, Cambridge University Press.

[16] Harris, C. (1985), Existence and Characterization of Perfect Equilibrium in Games of Perfect Information, Econometrica, 53, 613-628.

[17] Harris, C., Reny, P., Robson, A. (1995), The Existence of Subgame-Perfect Equilibrium in Continuous Games with Almost Perfect Information: A Case for Public Randomization, Econometrica, 63, $507-544$.

[18] Harsanyi, J.C., And Selten, R. (1988), A General Theory of Equilibrium Selection in Games, MIT Press.

[19] Hellwig, M. And Leininger, W. (1988), Markov-perfect Equilibrium in Games of Perfect Information, Discussion Paper A-183, University of Bonn.

[20] Himmelberg, C.J., Parthasarathy, T., Raghavan, T.E.S. and VAN VLECK, F.S. (1976), Existence of $p$-Equilibrium and Optimal Stationary Strategies in Stochastic Games, Proc. Amer. Math. Soc., $60,245-251$.

[21] Kohlberg, E. and Mertens. J.F. (1986), On the Strategic Sta- 
bility of Equilibria, Econometrica, 54, 1003-1037

1

[22] Levhari, D. and Mirman, L. (1980), The Great Fish War: An Example Using a Dynamic Cournot-Nash Solution, Bell J. Econ. 11, 322-334.

[23] Levy, Y. (2012A), A Discounted Stochastic Game with No Stationary Nash Equilibrium. DP \#596, Center for the Study of Rationality, Hebrew University, Jerusalem.

[24] Levy, Y. (2012B), A Cantor Set of Games with No ShiftHomogeneous Equilibrium Selection, Math. Oper. Res. (Forthcoming).

[25] Maitra, A. And Sudderth, W. (2007), Subgame-Perfect Equilibria for Stochastic Games, Math. Oper. Res., 32, 711-722.

[26] Maitra, A. and Parthasarathy, T. (1970), On Stochastic Games, J. Optim. Theory Appl., 5, 289-300.

[27] Maskin, E. And Tirole, J. (2001), Markov Perfect Equilibrium: I. Observable Actions, Journal of Economic Theory, 100, 191-219.

[28] Mertens, J.-F. and Parthasarathy, T.E.S. (1987), Equilibria for Discounted Stochastic Games, Paper 8750, Université Catholique de Louvain, Louvain-la-Neuve, Belgium.

[29] Monahan, G. And Sobel, M. (1994), Stochastic Dynamic Market Share Attraction Games, Games Econom. Behav., 6, 130-149.

[30] NowaK, A.S. (1985A), Universally Measurable Strategies in ZeroSum Stochastic Games, Ann. Probab., 13, 269-287.

[31] NowaK, A.S. (1985B), Existence of Equilibrium Stationary Strategies in Discounted Noncooperative Stochastic Games with Uncountable State Space, J. Optim. Theory Appl, 54, 591-602.

[32] NowaK, A.S. (2002) On a New Class of Non-Zero-Sum Discounted Stochastic Games having Stationary Nash Equilibrium Points, Internat. J. Game Theory, 32, 121-132.

[33] NowaK, A.S. (2007) Stochastic Games in Economics, Math. Methods 
Oper. Res, 66, 513-530.

1

[34] Nowak, A.S. and Raghavan, T.E.S. (1992), Existence of Stationary Correlated Equilibria with Symmetric Information for Discounted Stochastic Games, Math. Oper. Res., 17, 519-526.

[35] Parthasarathy, T. (1973), Discounted, Positive and Noncooperative Stochastic Games, Internat. J. Game Theory, 2, 25-37.

[36] Parthasarathy, T. (1982), Existence of Equilibrium Stationary Strategies in Discounted Stochastic Games, Sankhyā, 44, Ser. A, 114127.

[37] Phelan, C. and Stacchetti, E. (2001), Sequential Equilibria in a Ramsey Tax Model, Econometrica, 69, 1491-1518.

[38] Rogers, P.D. (1969), Nonzero-Sum Stochastic Games. Report ORC 69-8, Operations Research Center, University of California, Berkeley.

[39] Rosenthal, R.W. (1982), A Dynamic Model of Duopoly with Consumer Loyalties, J. Econ. Theory, 27, 69-76.

[40] Solan, E. (1998), Discounted Stochastic Games, Math. Oper. Res., 23, 1010-1021.

[41] Shapley, L. (1953), Stochastic Games. Proc. Natl. Acad. Sci. USA, 39, 1095-1100.

[42] Sobel, M. (1971), Noncooperative Stochastic Games. Ann. Math. Stat., 42, 1930-1935.

[43] Sobel, M. (1973), Continuous Stochastic Games. J. Appl. Probab, 10, 597-604.

[44] Simon, R. (2003), Games of Incomplete Information, Ergodic Theory, and the Measurability of Equilibria. Israel J. Math., 138, 1, 73-92.

[45] Takahashi, M. (1964), Equilibrium Points of Stochastic NonCooperative n-Person Games, Hiroshima J. Sci., 28, 95-99.

[46] Zachrisson, L. E. (1964), Markov Games, in M. Dresher, L. S. Shapley, and A. W. Tucker (eds.), Advances in Game Theory, Prince- 
1

2

3

5

6

7

9

10

11 ton, Princeton University Press, pp. 211-253.

2

3

4

5

6

7

8

9

10 